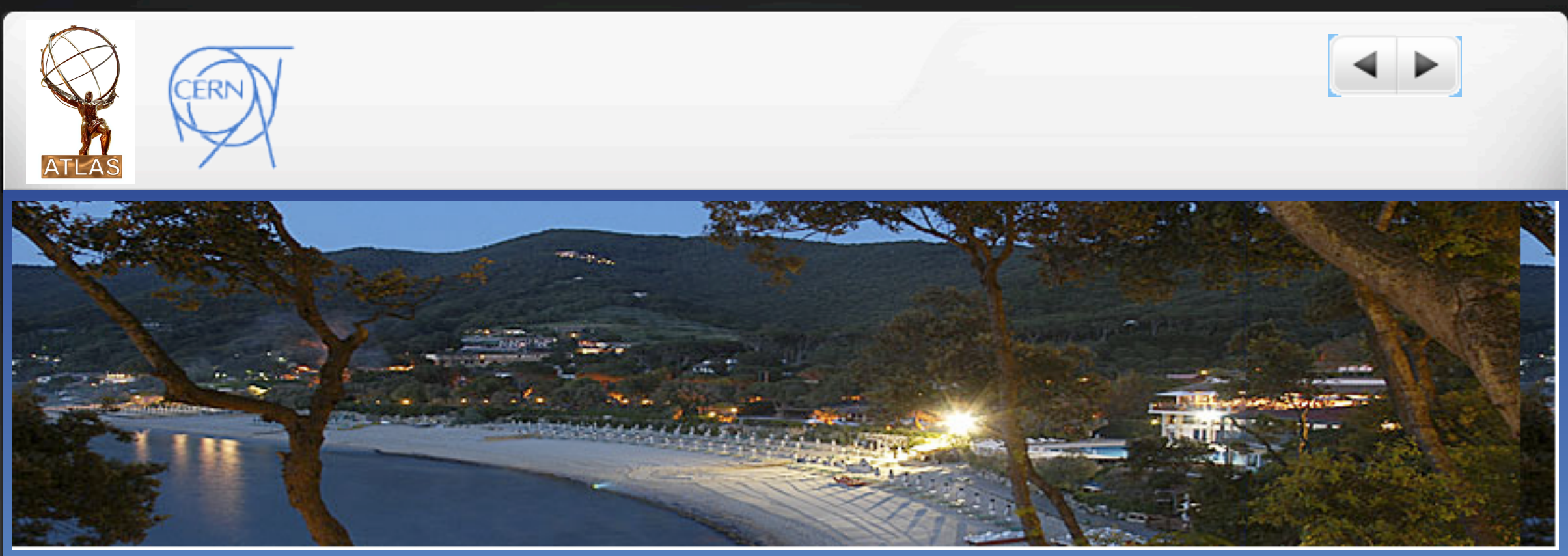

$1^{\text {th }}$ Pisa Meeting on Advanced Detectors

La Biodola, Isola d'Elba (Italy)

May 24 - 30, 2009

Readiness of the ATLAS detector : performance with the first beam and cosmic data

\author{
F.Pastore - CERN
}

On behalf of the ATLAS Collaboration 


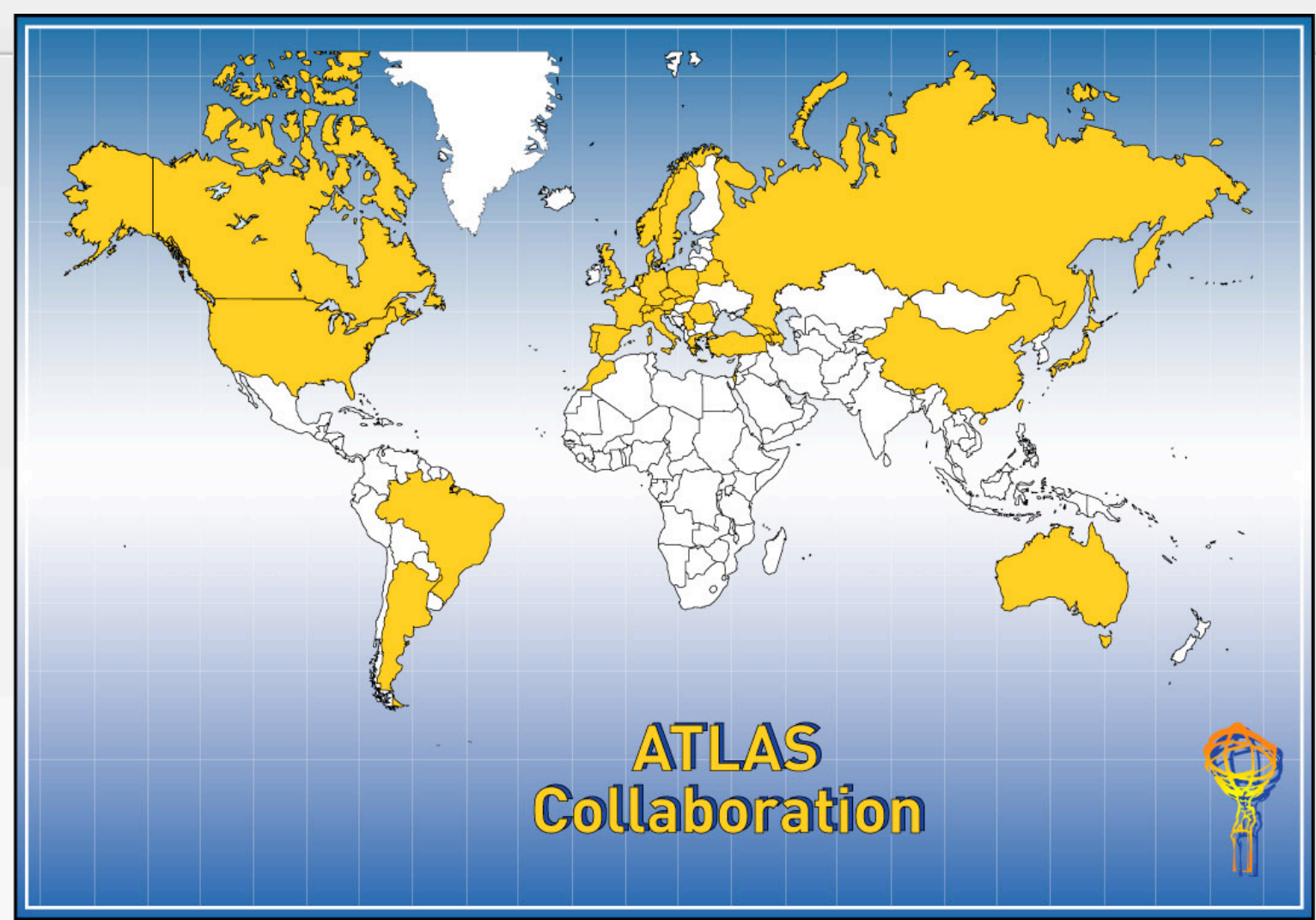

This talk is presented on behalf of the ATLAS Collaboration

- 37 Countries

- 169 Institutions

- 2500 Scientific Authors 
The ATLAS detector

- pp collisions @ $14 \mathrm{TeV}$ and $\mathrm{L}=10^{34} \mathrm{~cm}^{-2} \mathrm{~s}^{-1}$

- $\mathrm{LHC}$ collision rate $=40 \mathrm{MHz}$

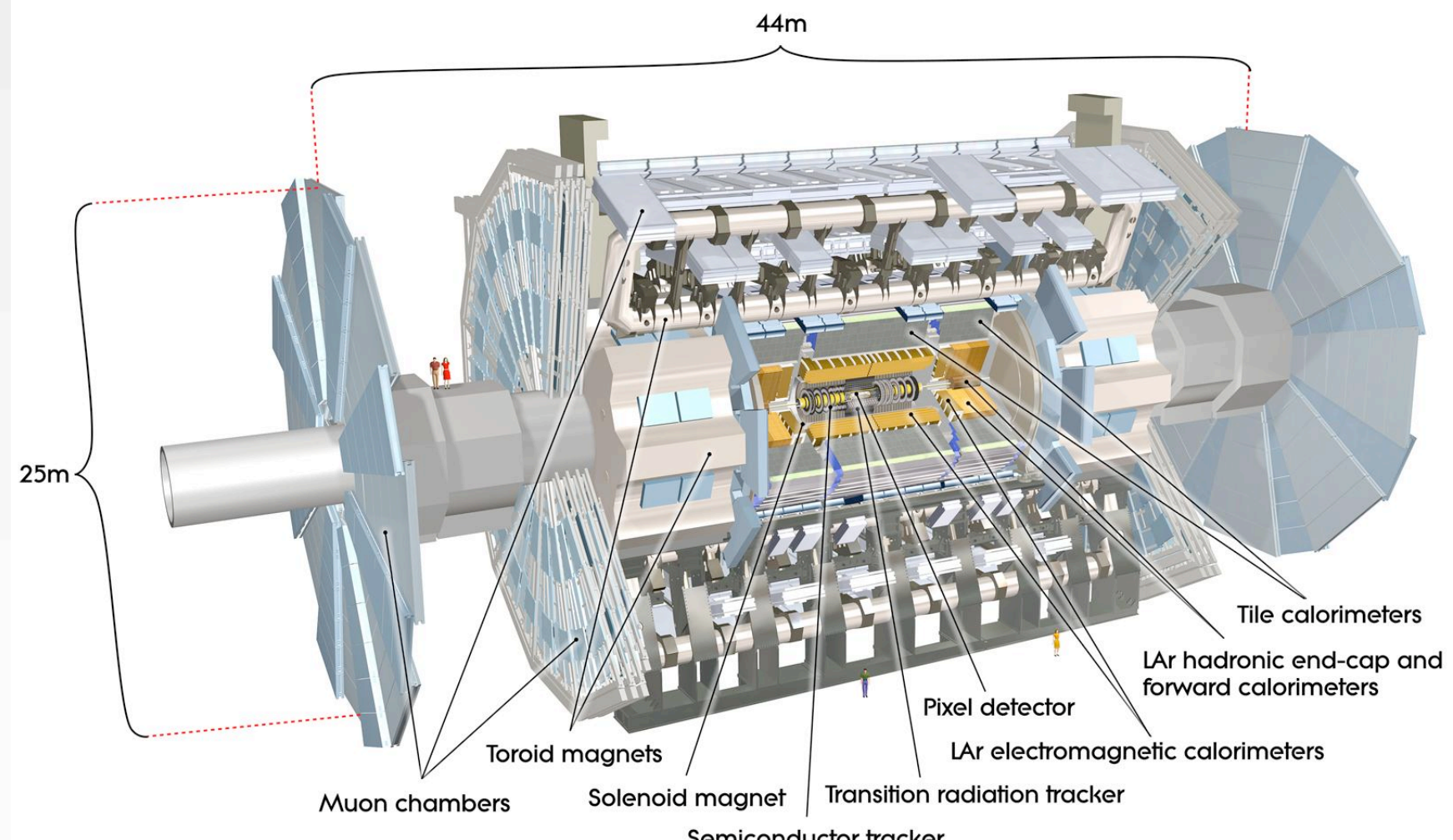

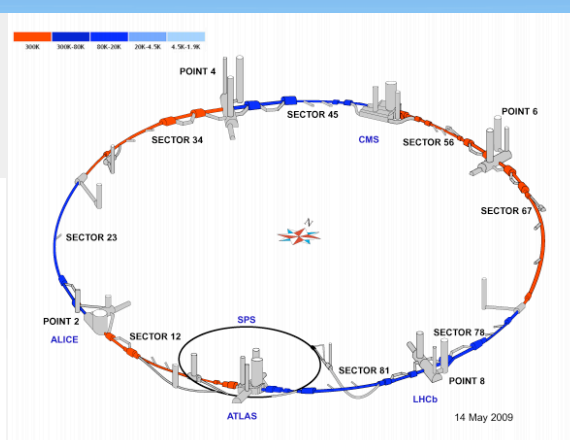




\section{Commissioning and readiness}

- The commissioning of the detector started in 2006, with consecutive Milestone and Technical Runs

- Operate together the various sub-systems even partially installed, testing global tools and infrastructure (DCS, DAQ, monitoring)

- DAQ performance and trigger systems

- Data transfer and processing (computing model and trigger selections)

- Results and integration of calibration tools and of track-based alignment

- On September $10^{\text {th }} 2008$, ATLAS was ready for operating continuously with the beams

- After the LHC accident, combined runs with cosmic rays continued

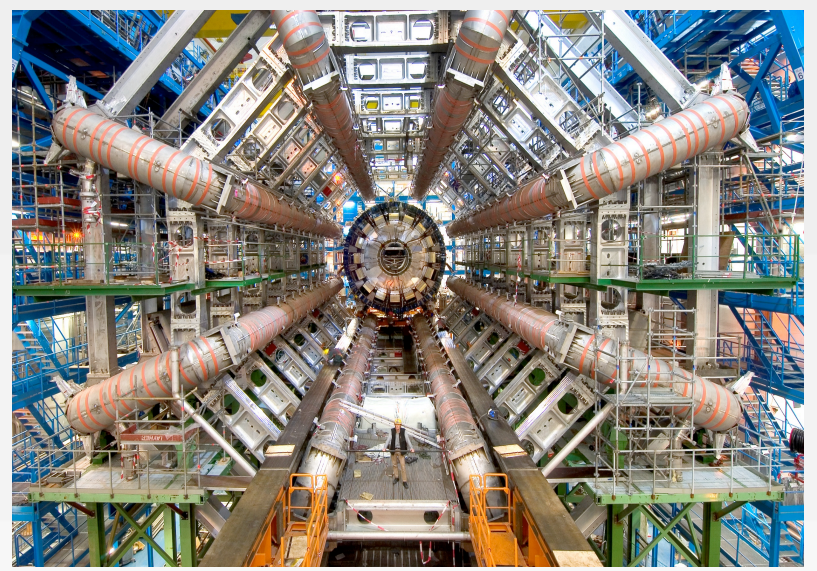




\section{Trigger and Data acquisition}

\section{Three-level trigger architecture, exploiting the "region-of-interest" (Rol) approach}

to reduce the data-flow requirements

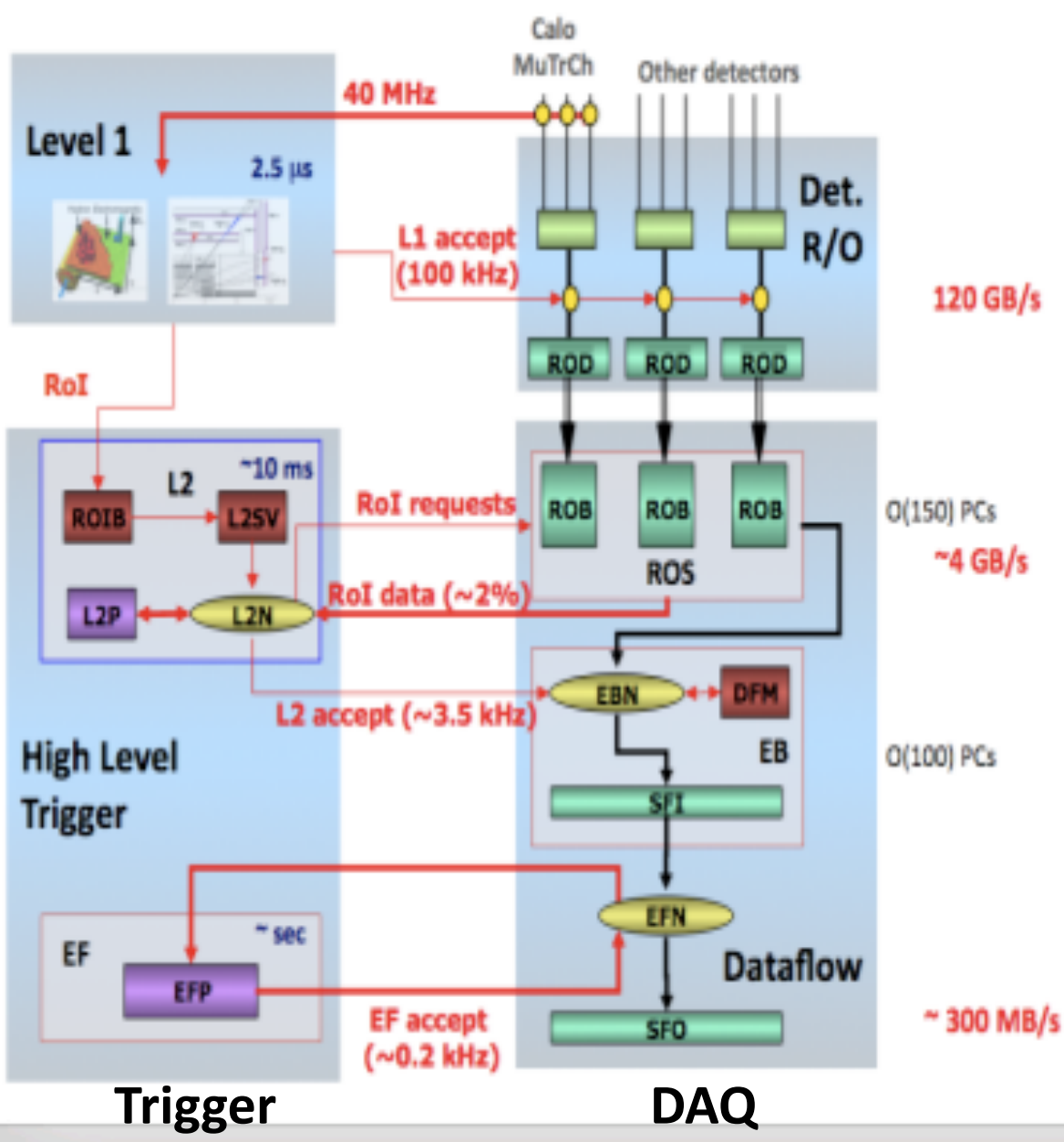

- Level-1 trigger (L1)

- Implemented in custom h/w

- Driven by local features in calorimeters and muons

- Rols in $\eta-\phi$ plane forwarded to Level 2

- Level-2 trigger (L2)

- Refines LVL1 results with a local analysis inside Rols

- Effective rejection mechanism that only requires collection of $1-2 \%$ of the event

- Lower requirements on the readout system (ROS) and network system

- Event filter (EF)

- Operates on fully assembled events

- Event building at $\sim 5 \mathrm{~GB} / \mathrm{s}$

- High Level Trigger (L2+EF)

- Keys: seeded reconstruction and early rejection

- Custom algorithms for L2, derived from offline for EF

- Same selection infrastructure

- Implemented on Linux PC farms 


\section{Trigger and Data acquisition: status}

- Level-1 trigger

- Tested up to $50 \mathrm{kHz}$, to be improved to nominal rate

- Timing-in in progress

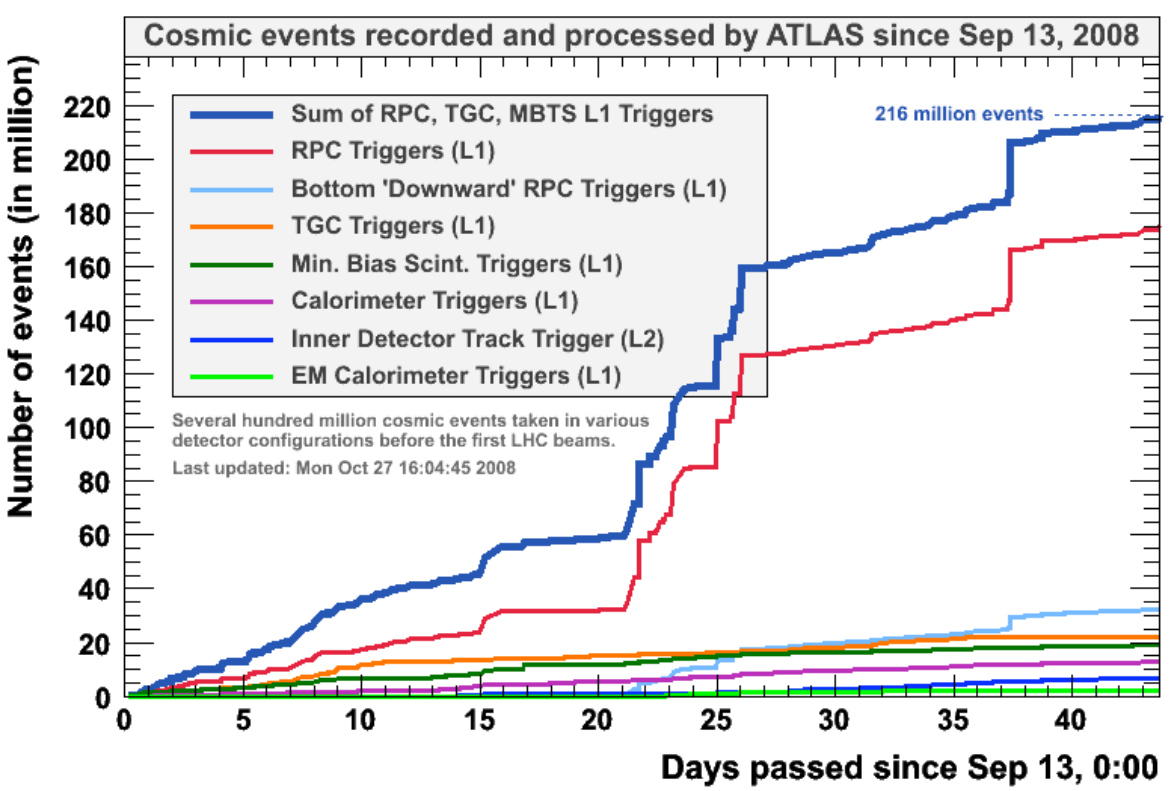

- High Level triggers (Level-2 and Event Filter)

- 850 HLT nodes (35\% final system) tested at $60 \mathrm{kHz}$

- Finalization of the system will be luminosity driven

- Expected: 500 Level-2 nodes + 1800 Event-Filter nodes (8 cores @ $2.5 \mathrm{GHz}$ )

- High-level algorithms (tracking) used during cosmic runs to enrich inner-detector samples

DAQ continuously running since 2008

- All ROS PCs installed and working

- Dataflow networking infrastructure fully in place

- Local storage farm (SFO) of 5 nodes with a total storage area of $50 \mathrm{~TB}$

Continuous cosmic data taking after September $12^{\text {th }}, 216$ millions of events, 453 TB of data, $400 \mathrm{k}$ files

- Realistic test of the computing model during summer 2008

- from online (SFO) to analysis at Tier-2 with simulated data @ L=1032 


\section{TDAQ performance}

- Dedicated technical runs to establish performance and scalability during system deployment

- Simulated data as input (background + signal)

- Test with $\mathrm{L}=10^{31}$ trigger menu

- $70 \%$ of $L 2,20 \%$ of EF

- Small event size: $800 \mathrm{kB}$, expected with LHC is $1.6 \mathrm{MB}$

- Stress test for the Data Acquisition system

- Stable running at $80 \%$ of designed L1 rate $(60 \mathrm{kHz})$

- Readout system (ROS) request rate up to $30 \mathrm{kHz}$

- Maximum expected is $20 \mathrm{kHz}$ ( $20 \%$ of L1)

- Event Building rate (L2-driven) : $4.2 \mathrm{kHz}(3.5 \mathrm{kHz}$ by design)

- Data storage (SFO) I/O rate: $550 \mathrm{MB} / \mathrm{s}$, with peak $>700 \mathrm{MB} / \mathrm{s}$

\section{ROS request EB and LVL2 Rates $(\mathrm{Hz})$}

during ATLAS combined cosmic run
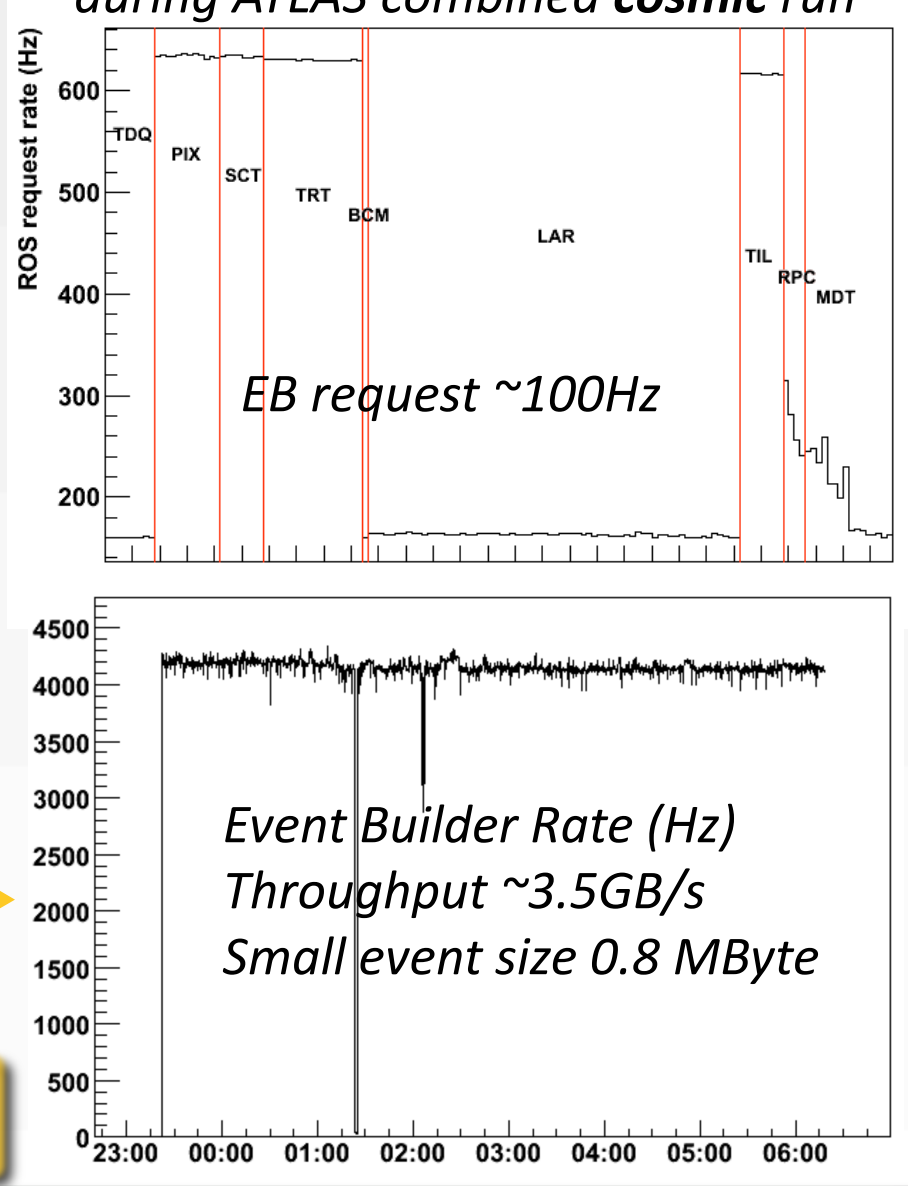

See D.Scannicchio talk at this conference 


\section{Detecting beams in ATLAS}

- ATLAS was "on" on Sep. 10th, although many components operated with reduced HV: SCT-barrel at very low bias, Pixel and CSC off (safety and late commissioning)

- In addition to Level-1 triggers, the beam can be detected by other systems

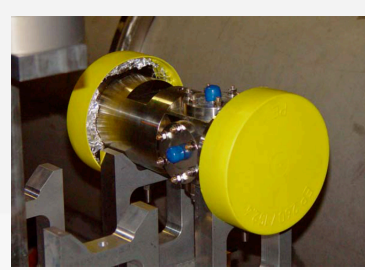

$175 \mathrm{~m}$

\section{Beam Pick-up}

Timing Experiment (BPTX)

- Provides a filled bunch trigger

- Monitor the beam activity: identifies the bunches in the beams, measures their individual intensity and phase relative to the clock, and assigns Bunch crossing ( $\mathrm{BC}=25 \mathrm{~ns}$ )

- Monitor of timing signals

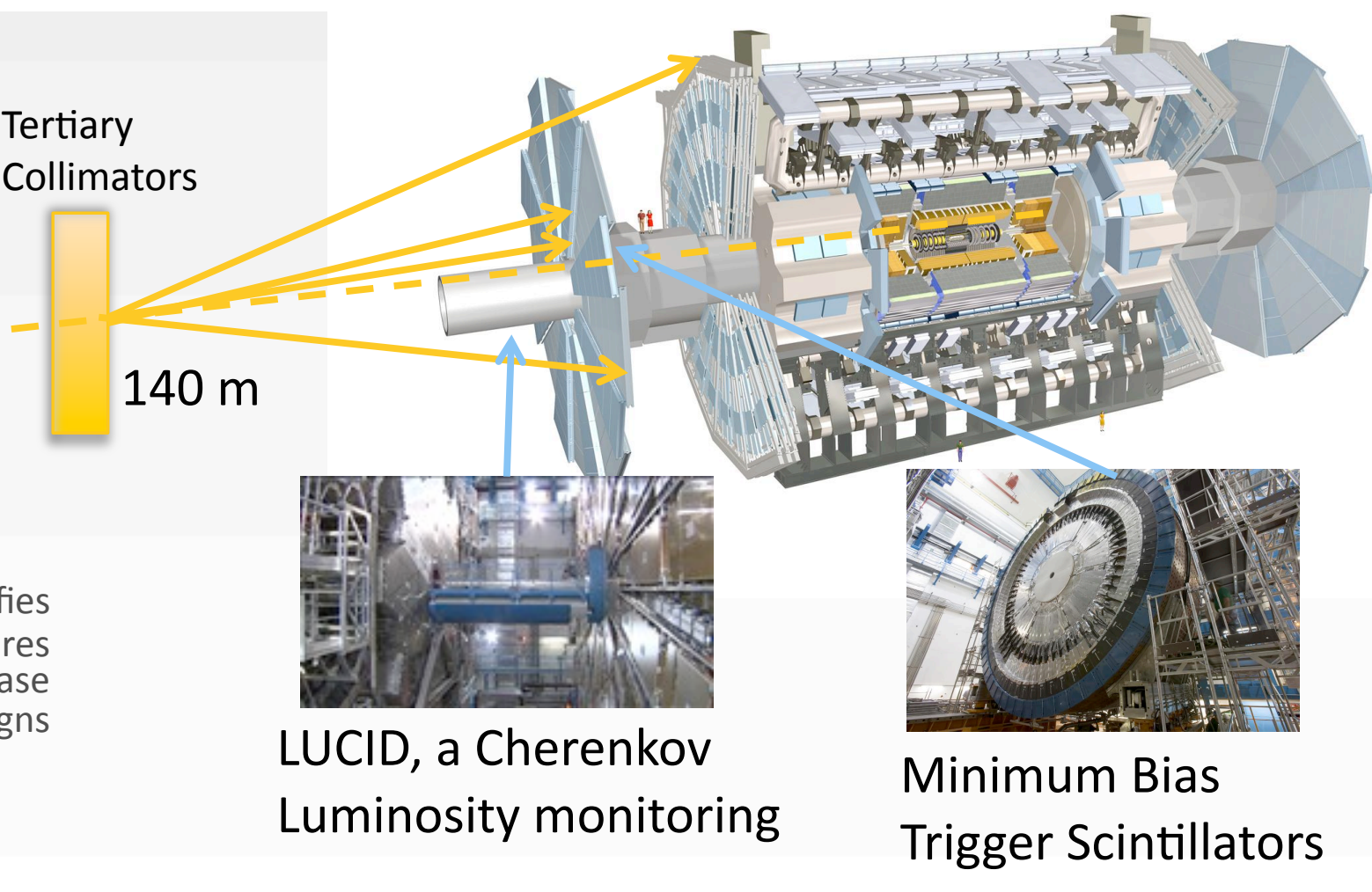




\section{Beams on $10^{\text {th }}-12^{\text {th }}$ September}

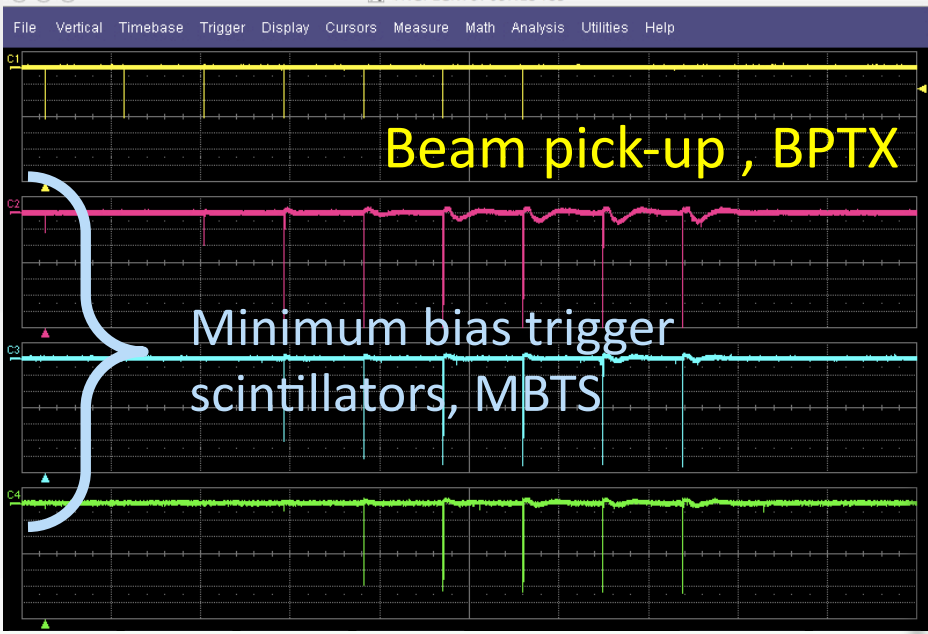

- Oscilloscope traces of discriminated signals during an injection of 1 bunch without RF capture

- The bunch manages to circulate a few times: after 7 turns its intensity falls below the threshold of the BPTX discriminator. The first few turns give only small activity in the MBTS, while after 3 or 4 turns they show saturated signals

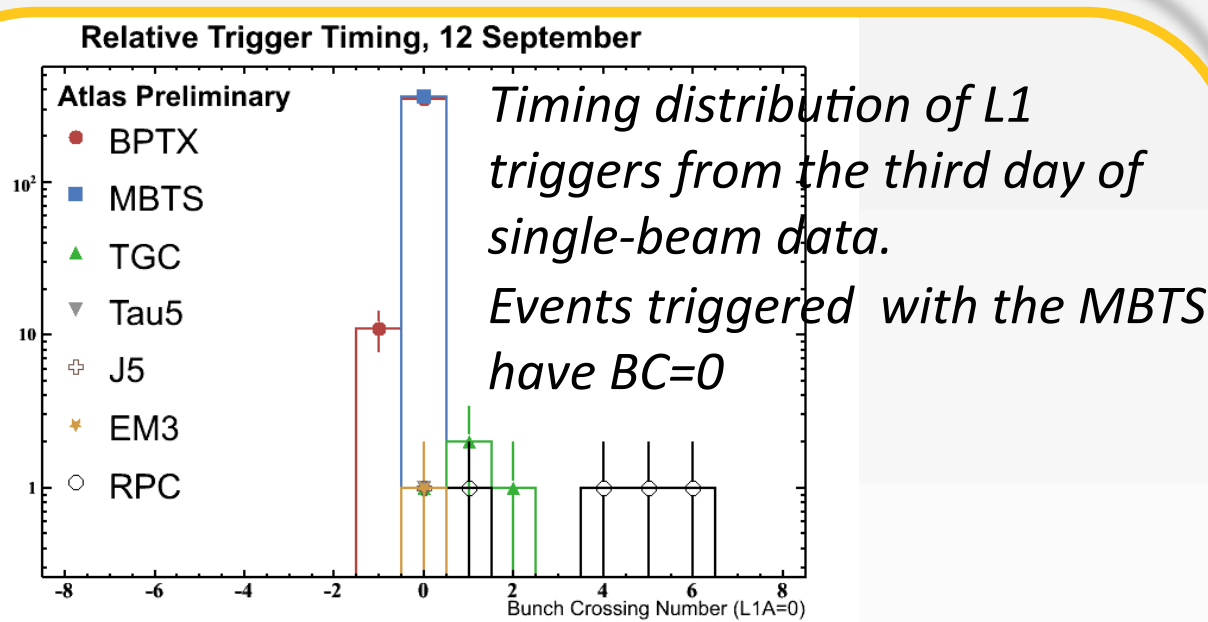

${ }_{0.15}^{0.2}$ Bunch intensity of beam 2

0.1 measured by BPTX - 20 minutes ${ }^{0.05}$ (10\% precision, energy not calibrated)

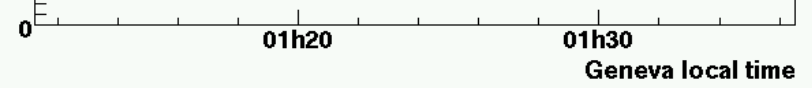

- Excellent timing of BPTX and MBTS

- Relatively small coincidence of MBTS with calorimeter and muon triggers - beam quality improved significantly in the first 48 hours 


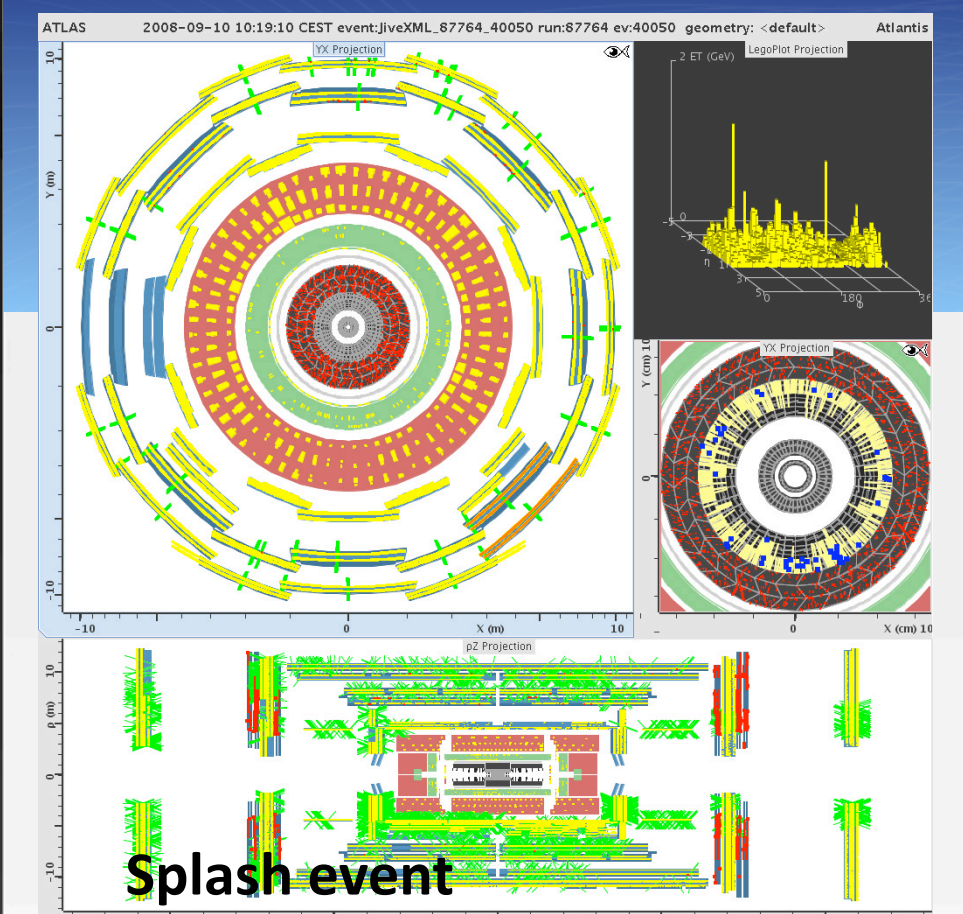

Some

LHC events

Beam halo events are important for

Endcaps calibrations
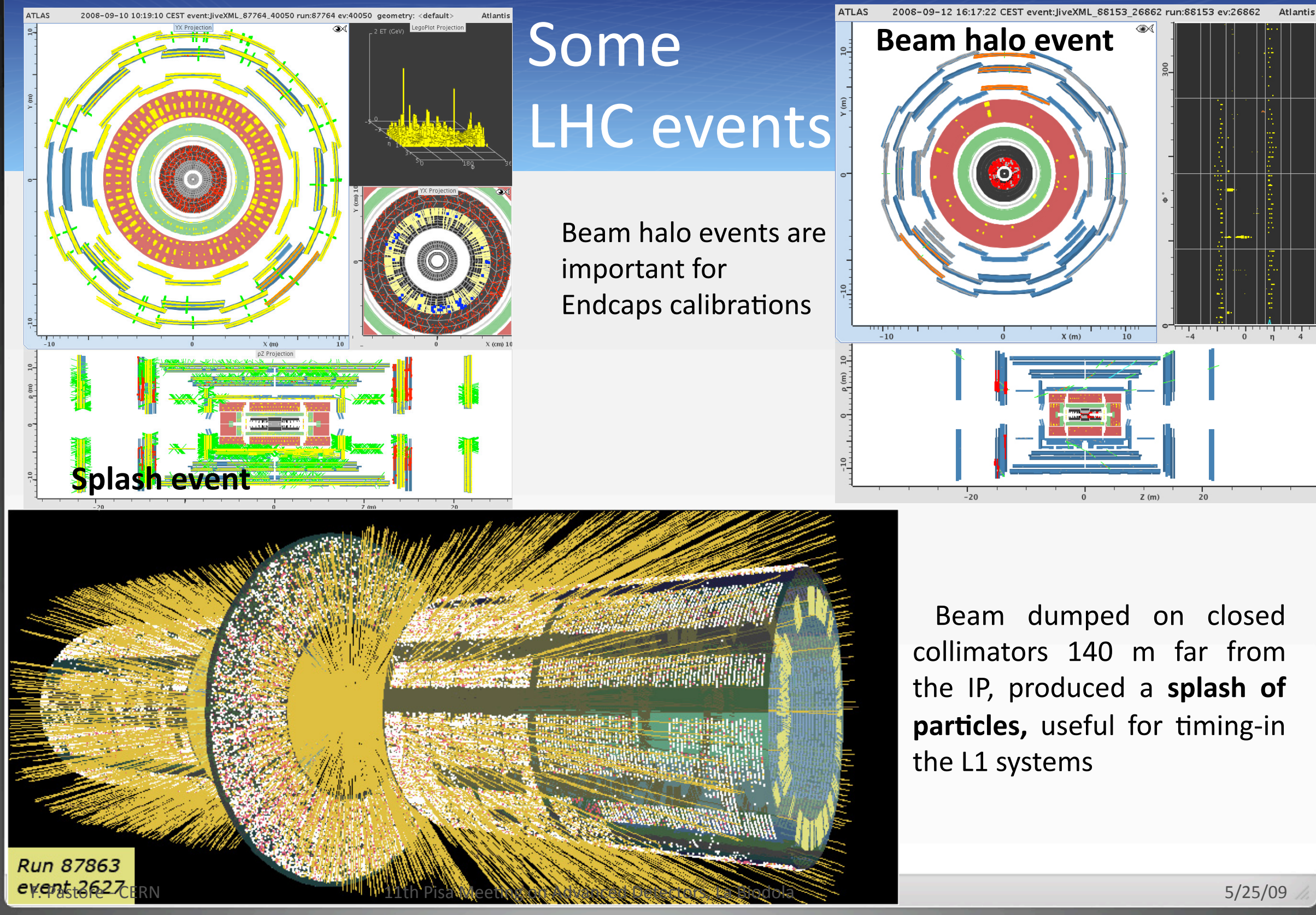

Beam dumped on closed collimators $140 \mathrm{~m}$ far from the IP, produced a splash of particles, useful for timing-in the L1 systems 


\section{ATLAS Magnet System}

\section{Barrel Toroid ( 8 coils)}

$L=25.3 \mathrm{~m}, \varnothing_{\text {outer }}=20 \mathrm{~m}$ $\mathrm{B}=0.2-2.5 \mathrm{~T}(@ 20.5 \mathrm{kA})$ Stored energy: $1100 \mathrm{MJ}$

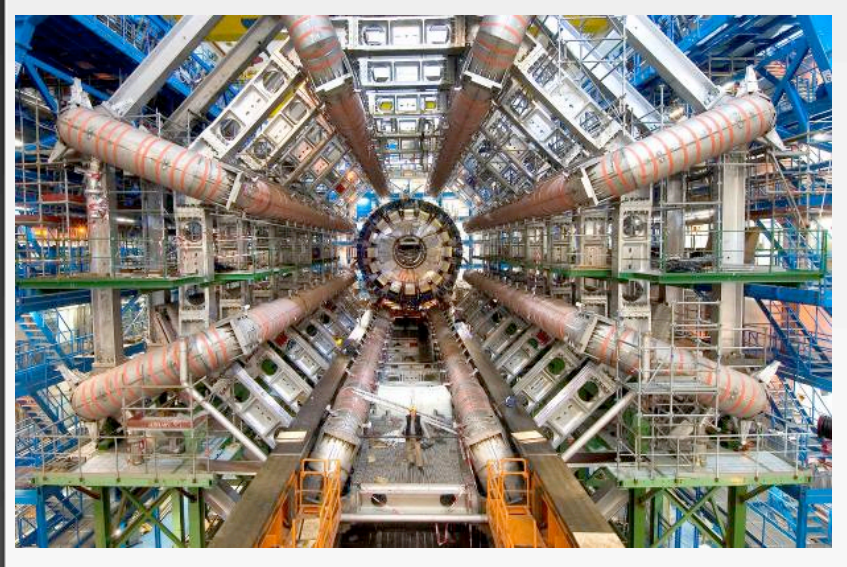

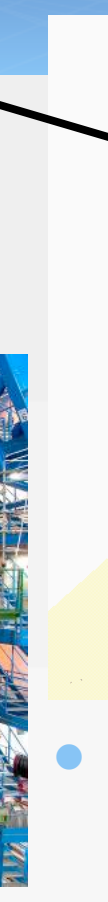

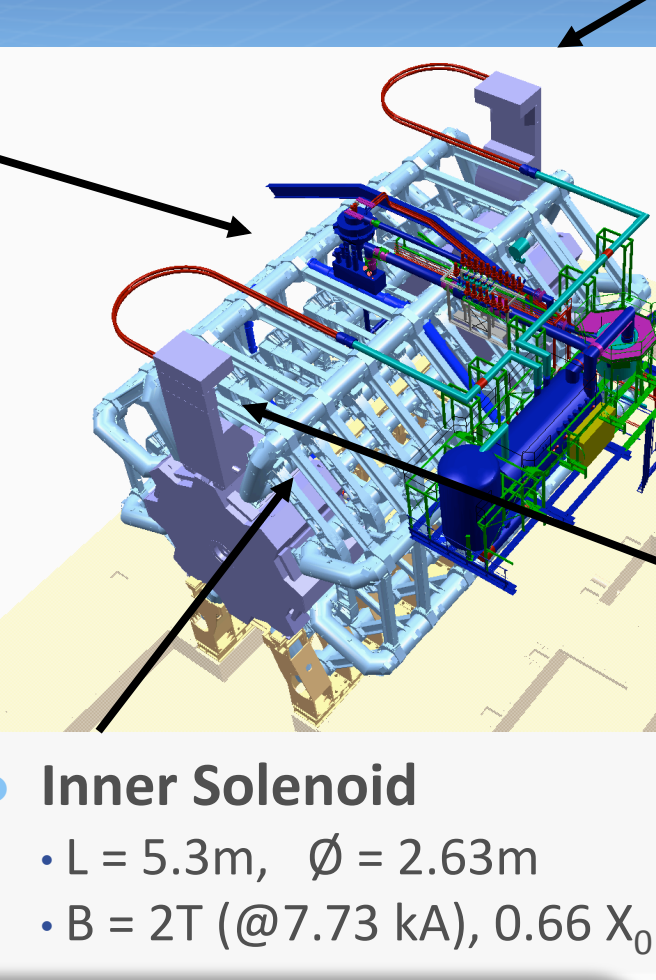

\section{Status}

- Stable continuous operation at nominal field

Stress and heat distributions during fast quenches are safe

Geometrical distortions of toroid with field-on as expected (measured by $\sim 1800$ Hall probes)

\section{Two Endcap Toroids}

8 coils common cryostat

$\mathrm{L}=5 \mathrm{~m}, \varnothing_{\text {outer }}=10.7 \mathrm{~m}$

$B=0.2-3.5 \mathrm{~T}(@ 20.5 \mathrm{kA})$

Stored energy: 250MJ
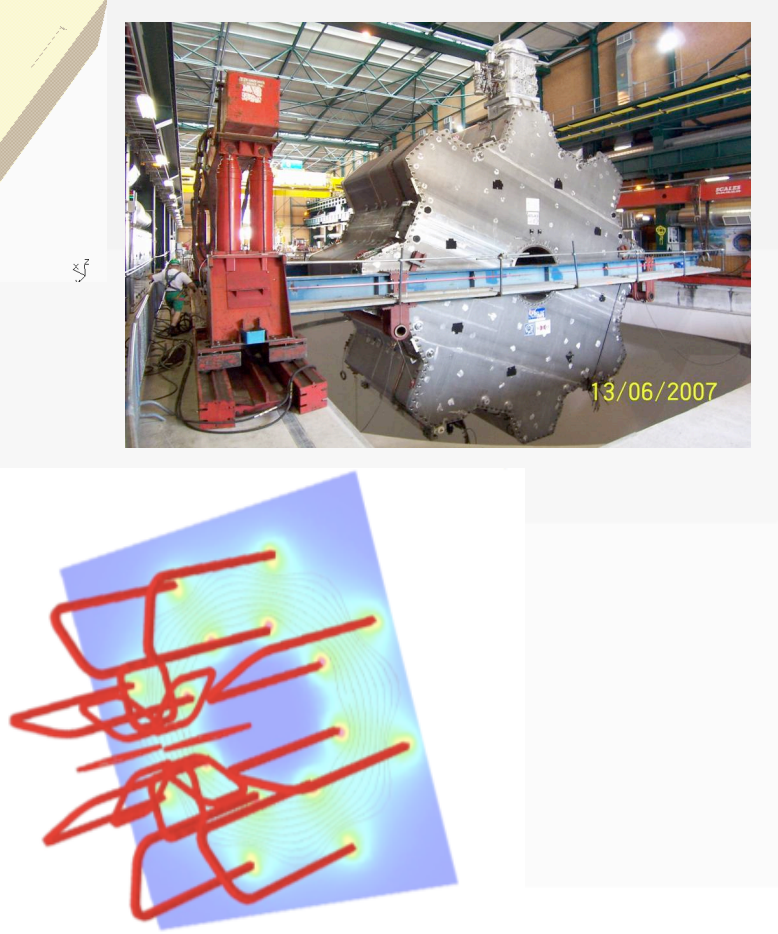


\section{Muon spectrometer}

Air-core toroid magnet system

Barrel: 1.5-5.5 Tm in $0<|\eta|<1.4$

Endcaps: 1-7.5 Tm in 1.6 $|\eta|<2.7$

Trigger chambers: $\boldsymbol{\eta}<\mathbf{2 . 4}$

Bunch-crossing ID, Muon pT triggering, second coordinate measurement
Precision chambers: $\boldsymbol{\eta}<\mathbf{2 . 5}$ Stand-alone $\mathrm{p}_{\mathrm{T}}$ measurement $\Delta \mathrm{p}_{\mathrm{T}} / \mathrm{p}_{\mathrm{T}}<10 \%$ up to $1 \mathrm{TeV}$
End-cap Trigger: Thin Gap Chambers (TGC)

-300k channels

$\sigma_{\mathrm{t}}<10 \mathrm{~ns}$

99.8\% operational dead channels $<0.01 \%$

noisy channels $<0.02 \%$
Barrel Trigger: Resistive Plate Chambers (RPC)

360k channels

$\sigma_{\mathrm{t}} \sim 2 \mathrm{~ns}$

Monitored drift tubes (MDT)

See D.Orestano talk at this conference

\section{Monitored Drift Tubes (MDT)}

1088 chamber with 339k channels 99.8\% operational

dead channels $<0.1 \%$

noisy channels $<0.2 \%$
95.5\% operational dead strips $<2 \%$ hot strips $<1 \%$

F. Pastore - CERN
Cathode strip Chambers (CSC)

Small Wheel End-cap

$2 \mathrm{D}$ readout

$100 \%$ operational

dead channels $<0.1 \%$ 


\section{Precision chambers (MDT) performance}

\section{Expected resolution}

- single tube $~ 80 \mu \mathrm{m}$

- 3-4 tubes $\sim 50 \mu \mathrm{m}$

- calibrations and alignment better than $30 \mu \mathrm{m}$
- MDT tubes are calibrated using muon tracks through iterative auto-

calibration procedure. To enlarge the muon sample, data extracted from L2 are sent to calibration centers (Tier-2) for extracting t $0, r$-t relations

- Residuals calculated after applying $t_{0}$ refit on single chamber timing spectra (cosmic approach to limit trigger jitter effects)

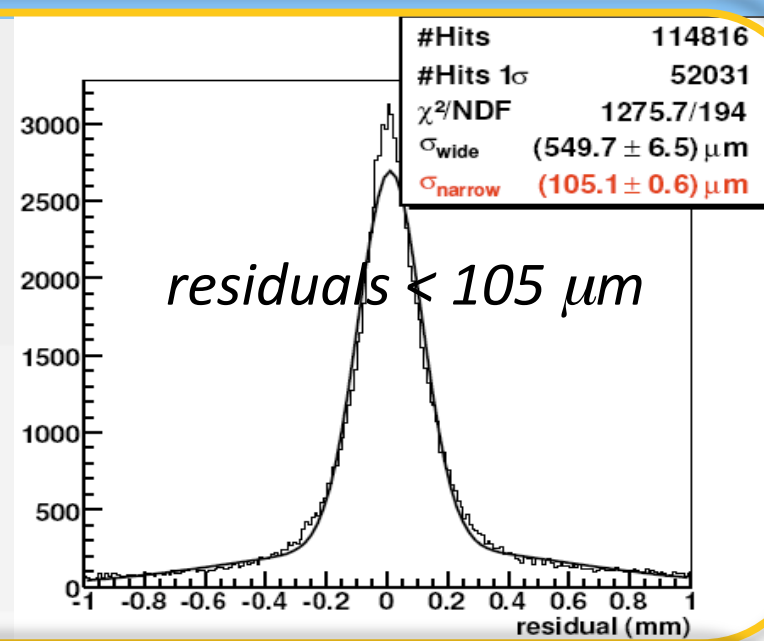

\section{See I.Potrap poster at this conference}

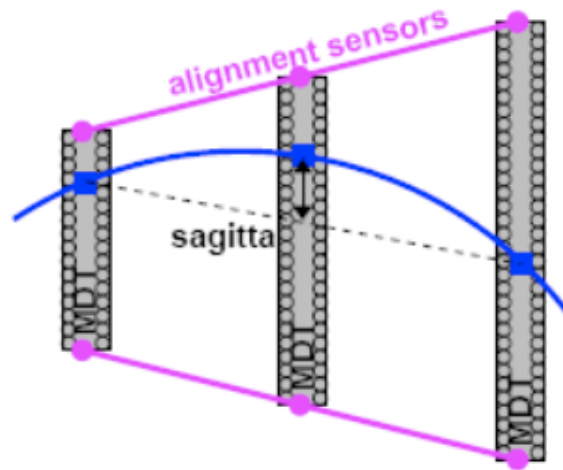

- Track sagitta calculated with the measured chamber position confirms the optical alignment system performance (45-50 $\mu \mathrm{m})$

- End-Caps, distributions are centered at 40 um

- Large Barrel chambers at 200 um level

- Small Barrel chambers (no direct projective lines) still at the $1 \mathrm{~mm}$ level.

\section{2 optical sensors}

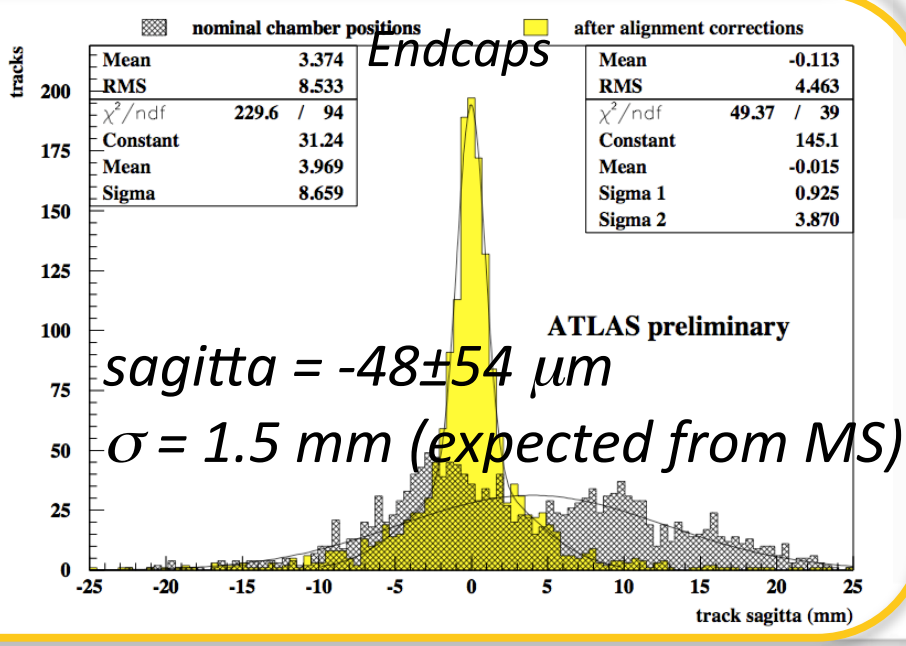




\section{Muon trigger chambers}

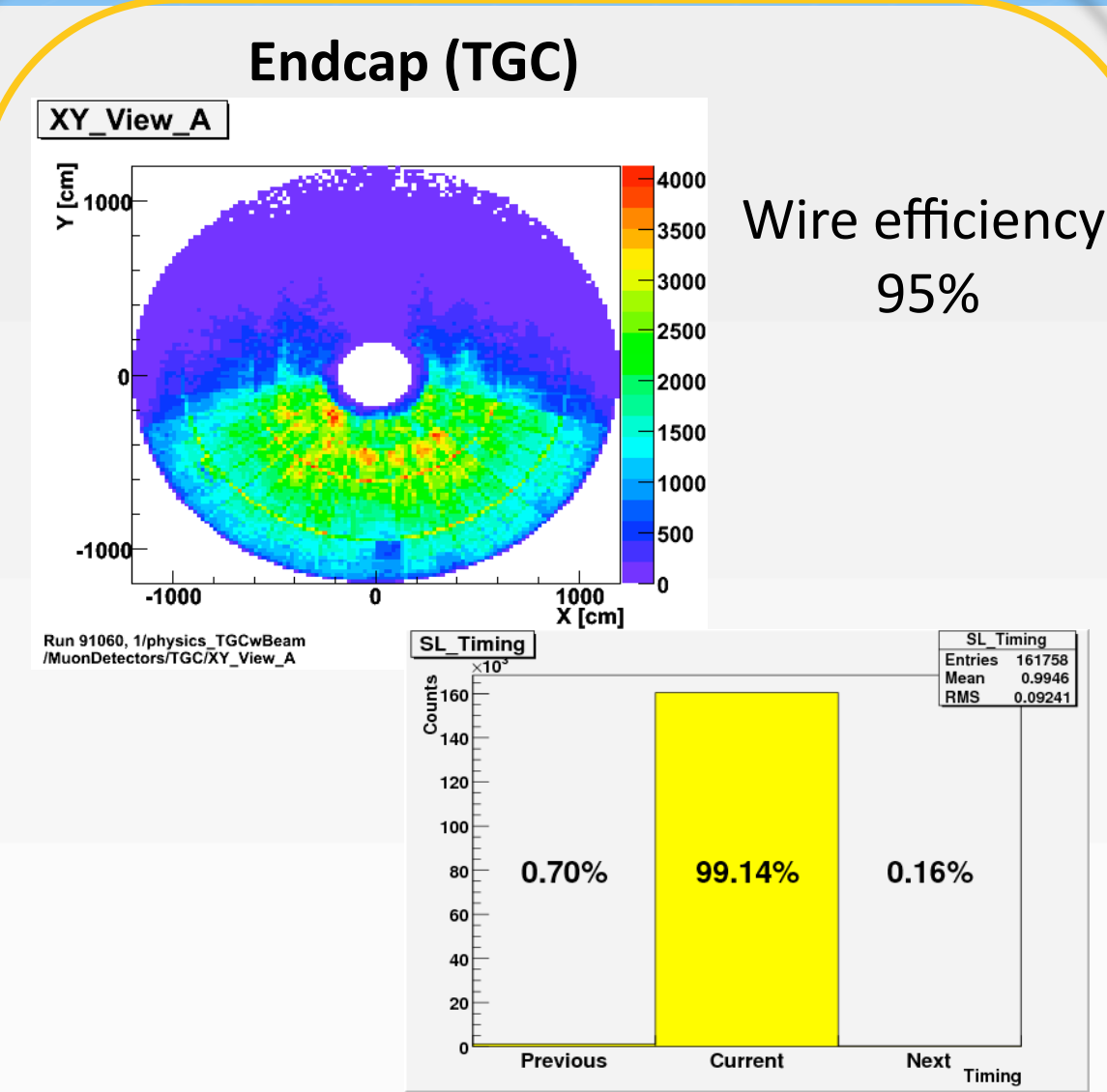

More than $99 \%$ of the trigger events are within the correct $B C$
Barrel (RPC)

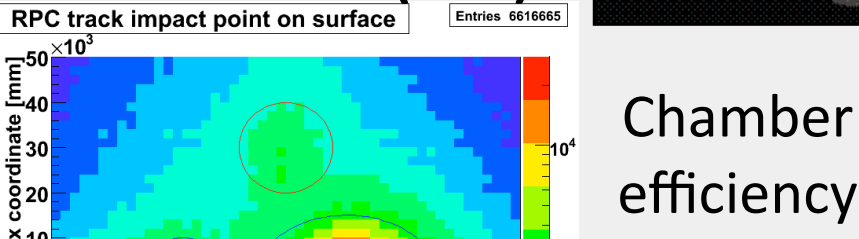

$97 \%$

Time difference between triggering ${ }^{0.6}$ layers RMS $=5.5 \mathrm{~ns}$

Local time alignment is

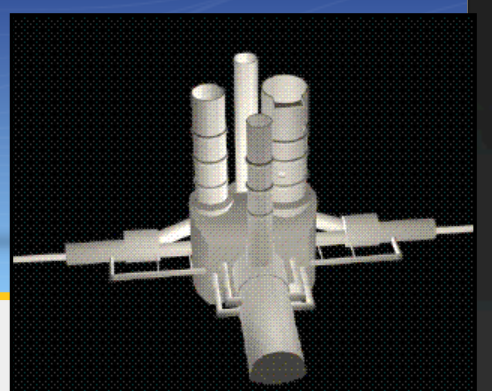

Chamber 


\section{Calorimeters}

\section{Liquid Argon:}

- Electromagnetic (Pb-LAr)

- accordion geometry

- 3 longitudinal segments

- Pre-shower for $\mathrm{h}<1.8$

- Hadronic (Cu/W-LAr)

- 4/3 longitudinal samples

- Status

- Dead channels: $<1 \%$ recoverable

- Noisy channels: $0.003 \%$

- Electronic calibration operational

Fe - Scintillation Tiles (barrel Hadronic)

- Dead channels $<1.4 \%$ under repair

- Calibration procedure operational (CS source, laser, charge injection)

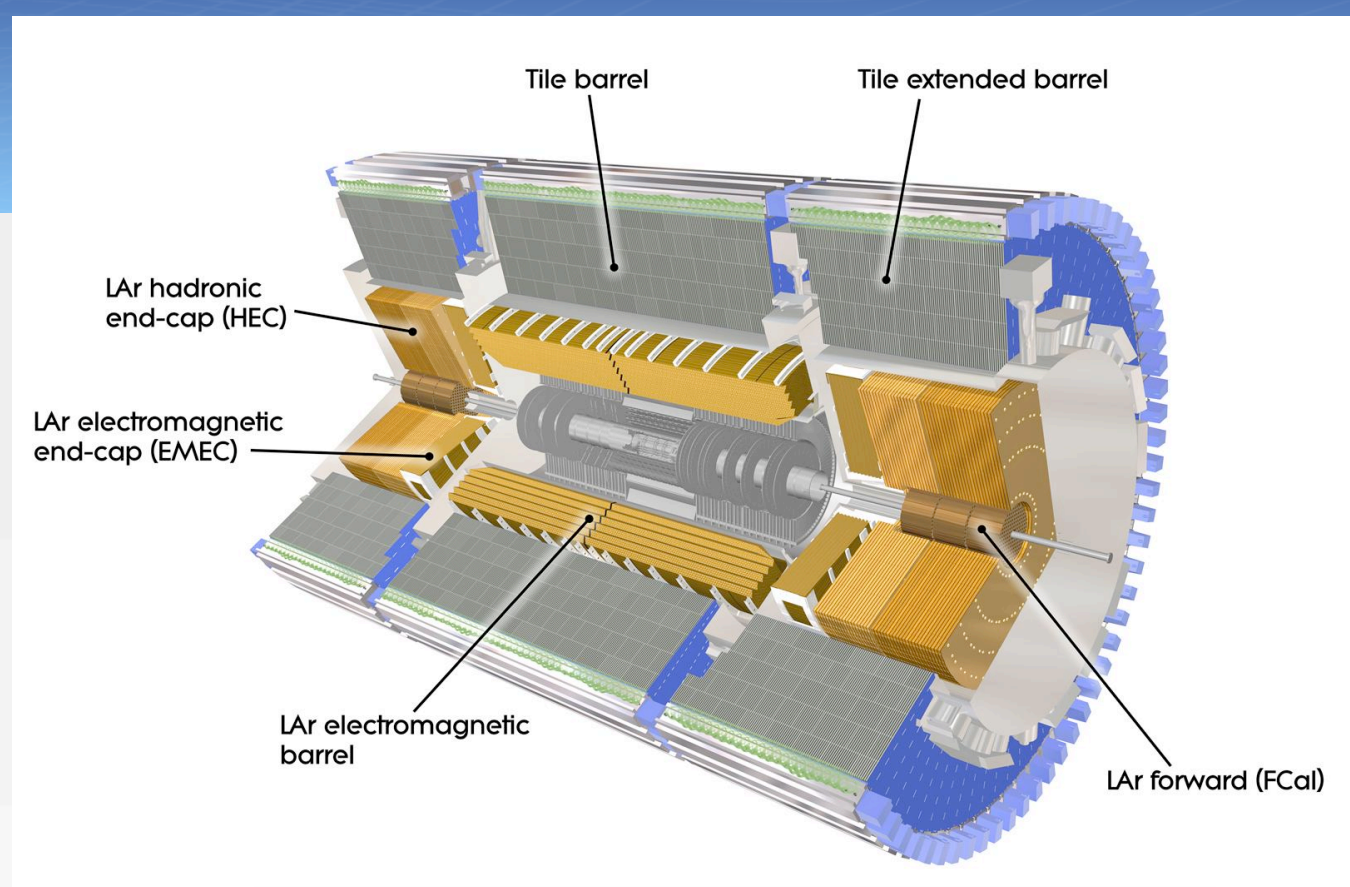

Electromagnetic energy resolution:

$\sigma(E) / E=10 \% / \sqrt{E} \oplus 0.7 \%$

Hadronic energy resolution:

$\sigma(E) / E=50 \% / \sqrt{E} \oplus 3 \% \quad(\eta<3.2)$

$\sigma(E) / E=100 \% / \sqrt{E} \oplus 10 \%(\eta>3.1)$

-Precision measurement of $e / \gamma$ for $\eta<2.5$

- Linearity to $0.1 \%$ 


\section{Liquid Argon (LAr) performance}

- Very stable pedestals

- Timing is already set to within a few ns for real collisions.

Survey of 128 channels in EM Front Layer

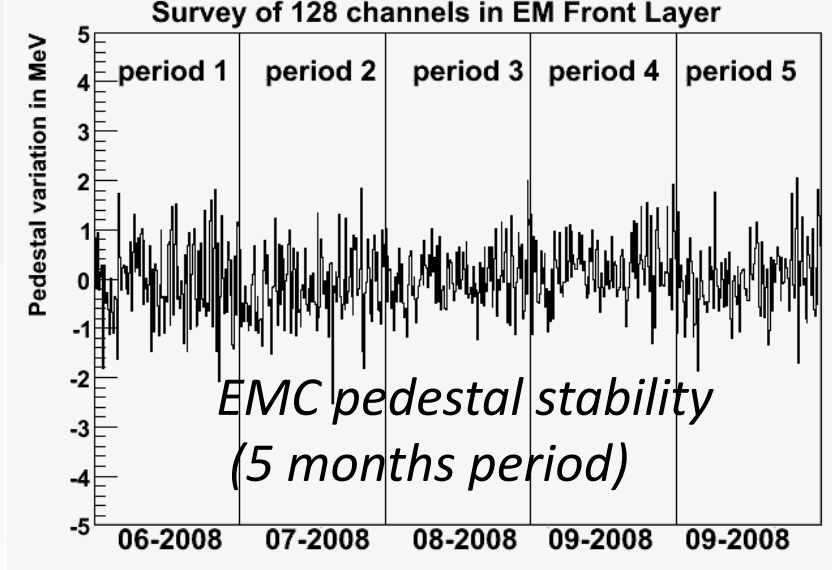

See S.Laplace talk at this conference

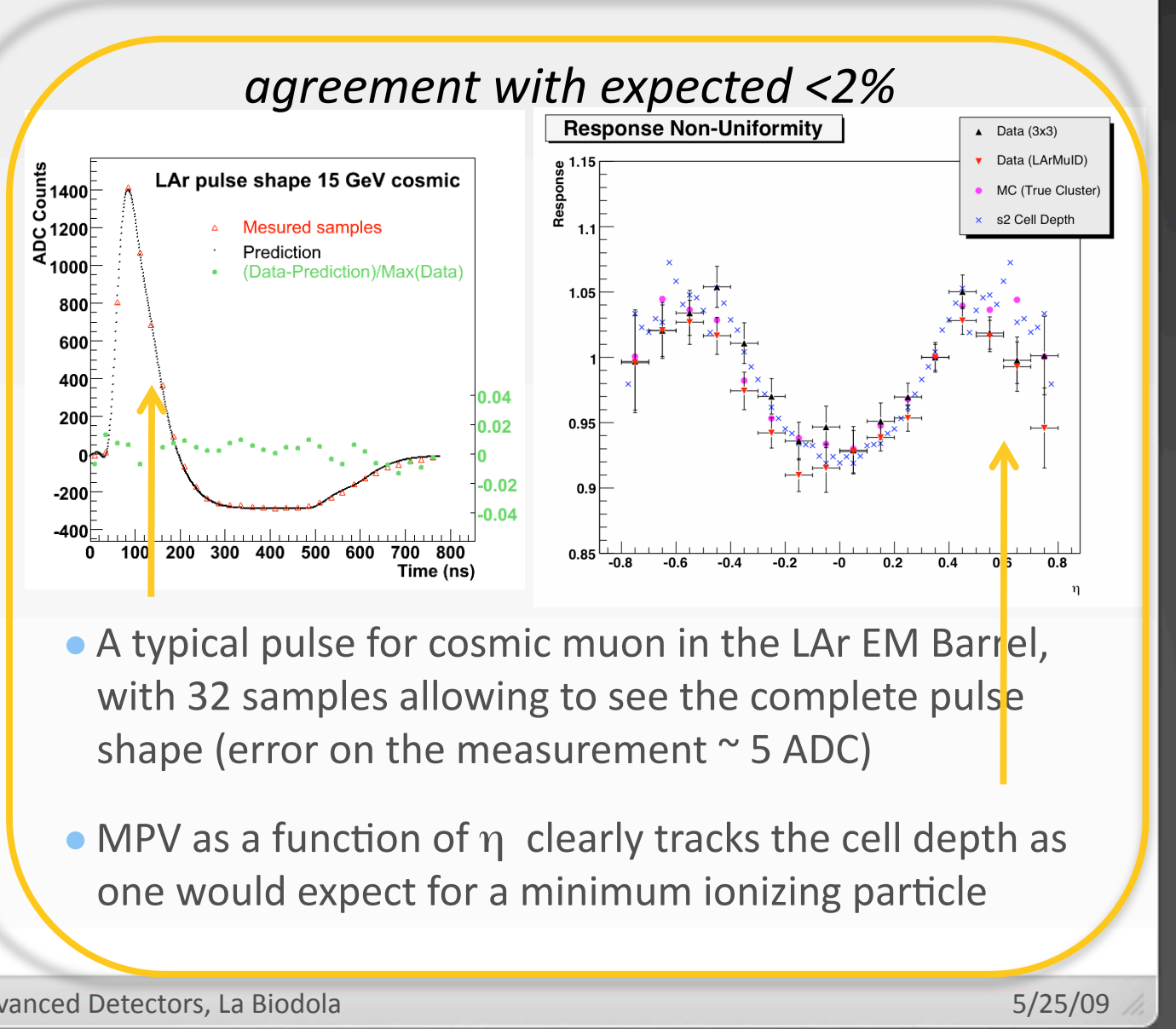




\section{Tile performance}

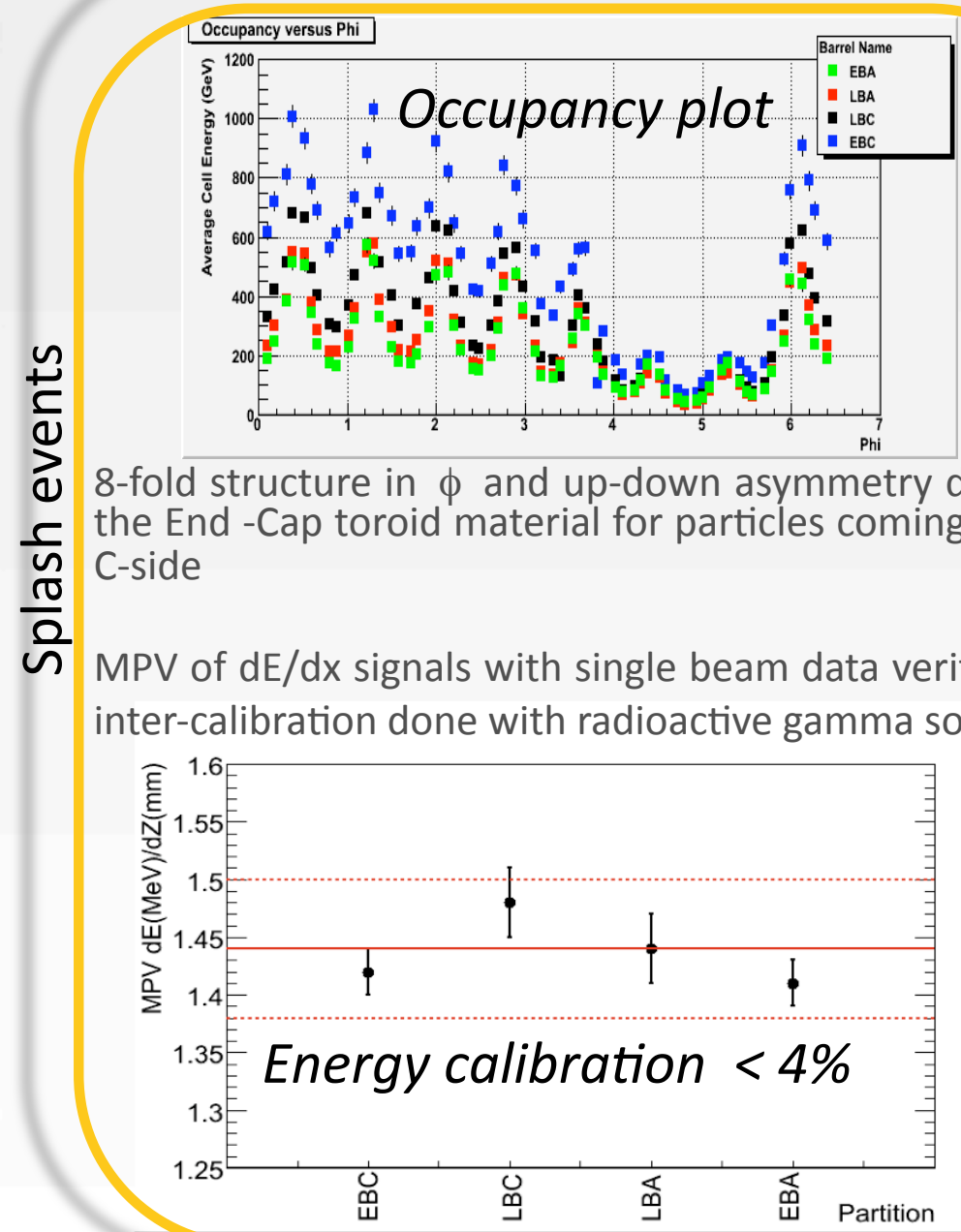

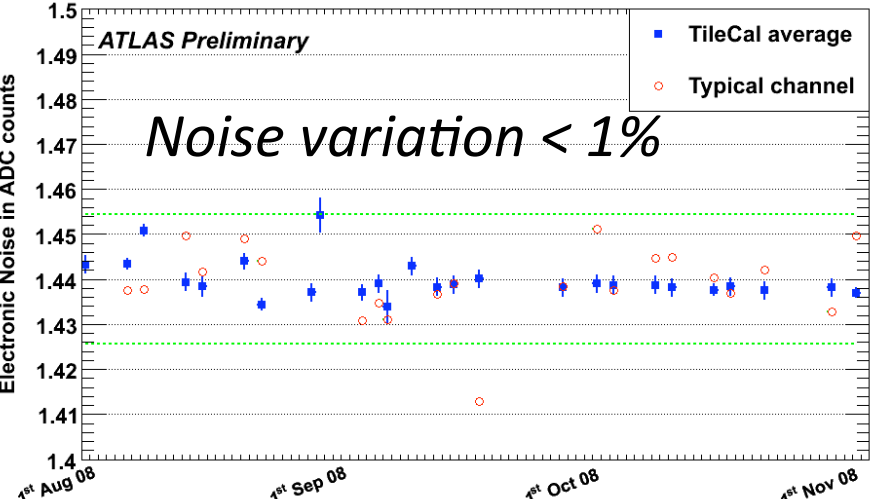

Stability in time of electronic noise (RMS of the digital samples) at cell-level (crucial for reconstruction)

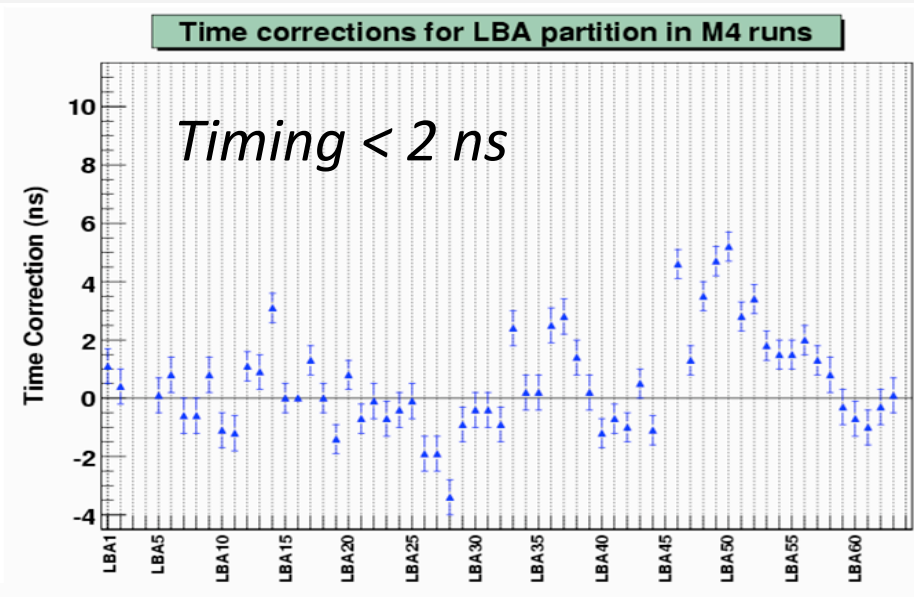

The time difference between measured and expected time-of-flight confirms the Laser time calibration 


\section{L1 Calorimeter trigger}

- Energy correlation between trigger towers (mainly $0.1 \times 0.1$ in $\eta / \phi$ ) and the full calorimeter readout (LAr), with nominal calibration

Splash event: the colors are proportional to the transverse energy deposited in each trigger tower
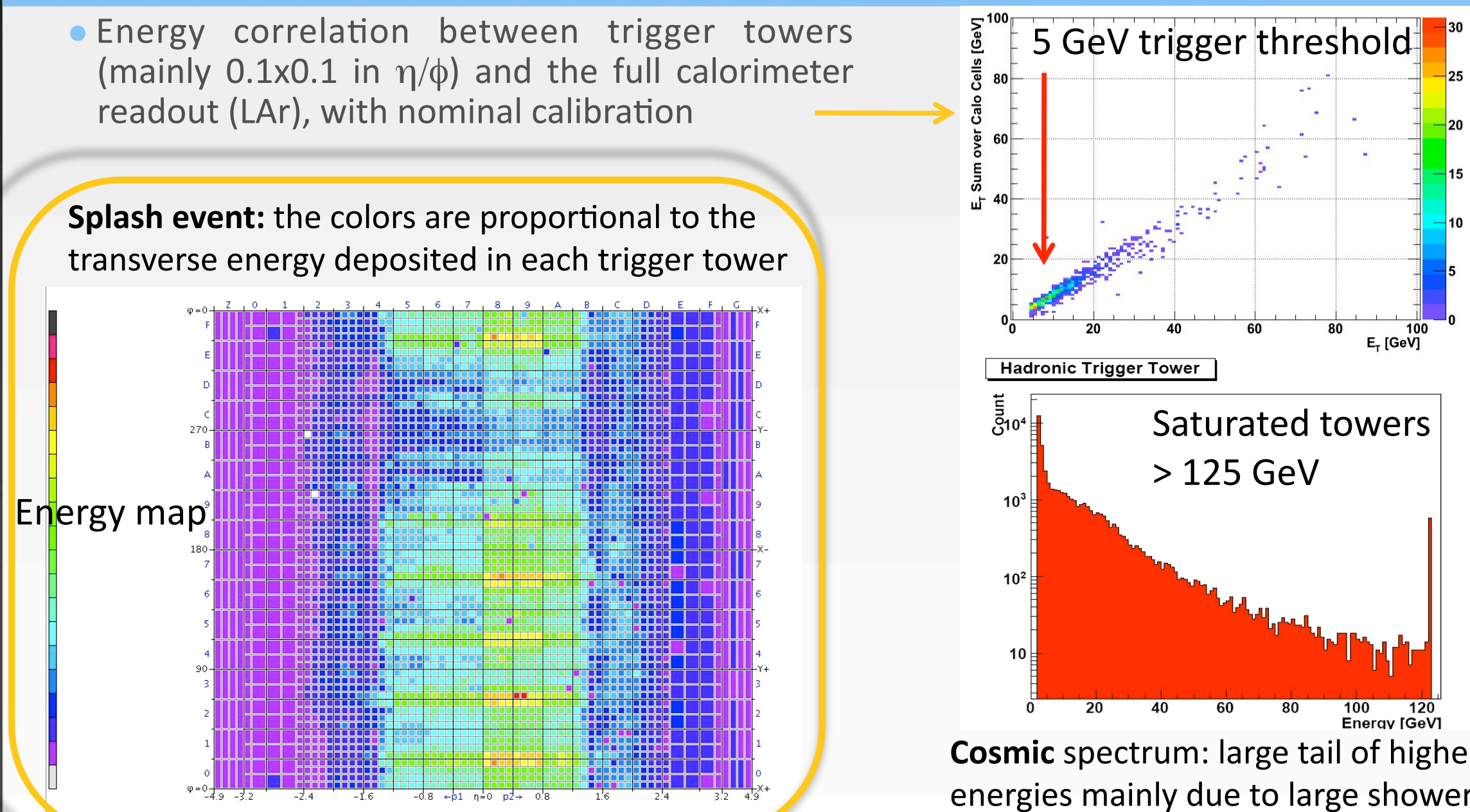

Cosmic spectrum: large tail of higher energies mainly due to large showers 


\section{Inner Detectors}

\section{Silicon Pixel detectors}

- pixel size $50 \times 400 \mu \mathrm{m}^{2} ; 80 \mathrm{M}$ channels

$\stackrel{\times}{\mathrm{d}}$ resolution: $10 \mu \mathrm{m} \times 110 \mu \mathrm{m}$

$>\quad>95 \%$ of modules operational

Noise occupancy: $5 \times 10^{-10}$; hit- $\varepsilon$ : $98 \%$

$\forall$ Semiconductor Tracker (SCT)

¿ $\quad 80 \mu \mathrm{m}$ strip width; 6M channels

竞 Resolution: $17 \mu \mathrm{m} \times 580 \mu \mathrm{m}$

ज्ञ $\quad>99 \%$ of barrel and $97 \%$ of end-cap

¿ modules operational

Noise: $<5 \times 10^{-5}$; hit- $\varepsilon>99 \%$

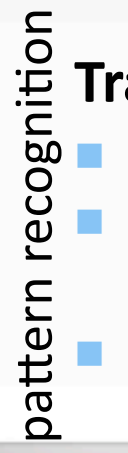
ID in $0.5<E<150 \mathrm{GeV}$

$98 \%$ of channels operational

$4 \mathrm{~mm}$ diameter straw tubes

Up to 36 points for tracking and $e / \pi$
Operated inside the 2 Tesla magnetic field

$\eta<2.5$ (TRT: $\eta<2.0) \quad \sigma / p_{T}=\left(0.05 p_{T}+1\right) \%$

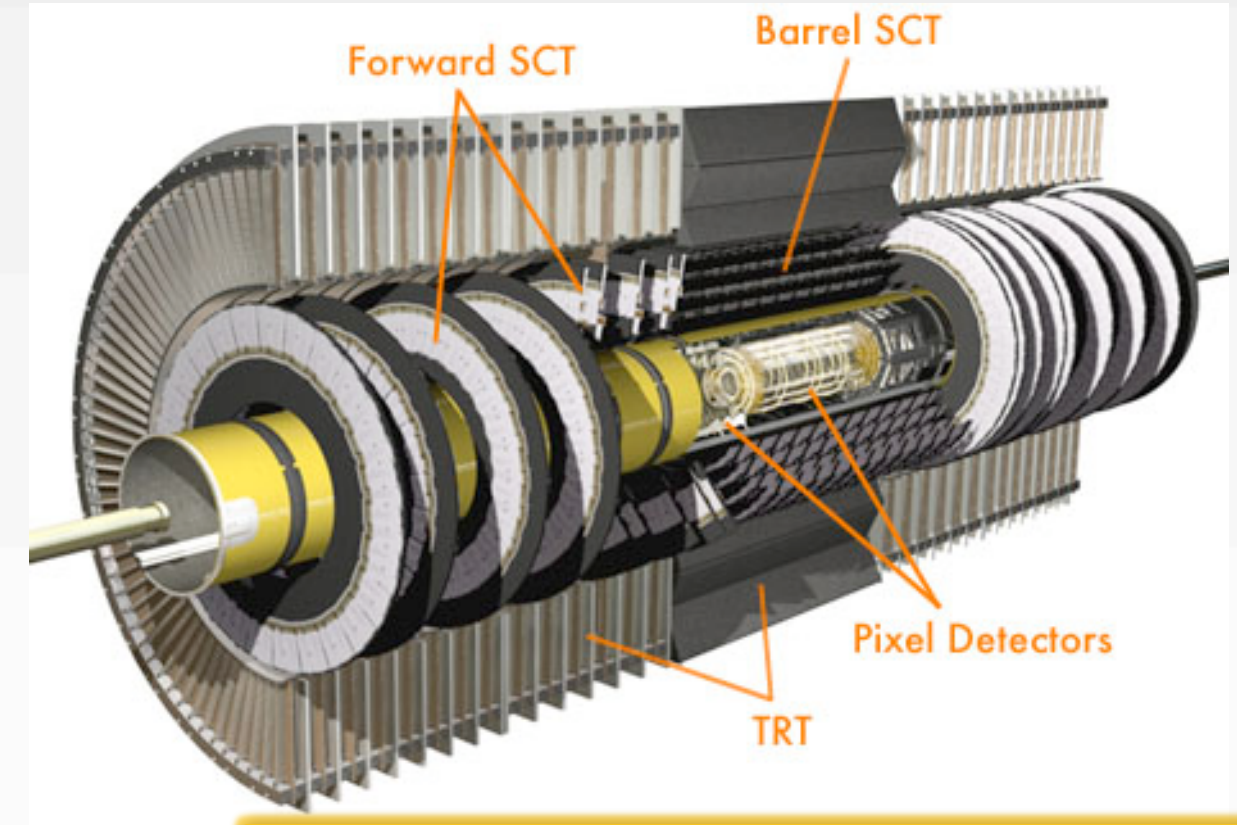

See A.Andreazza talk at this conference

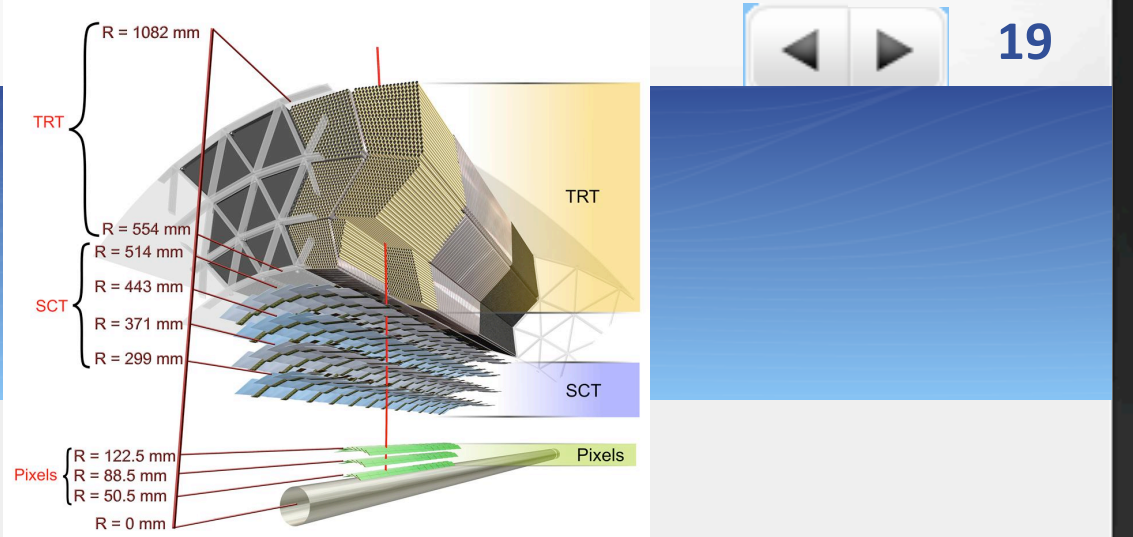




\section{Beam splash events seen in the ID}
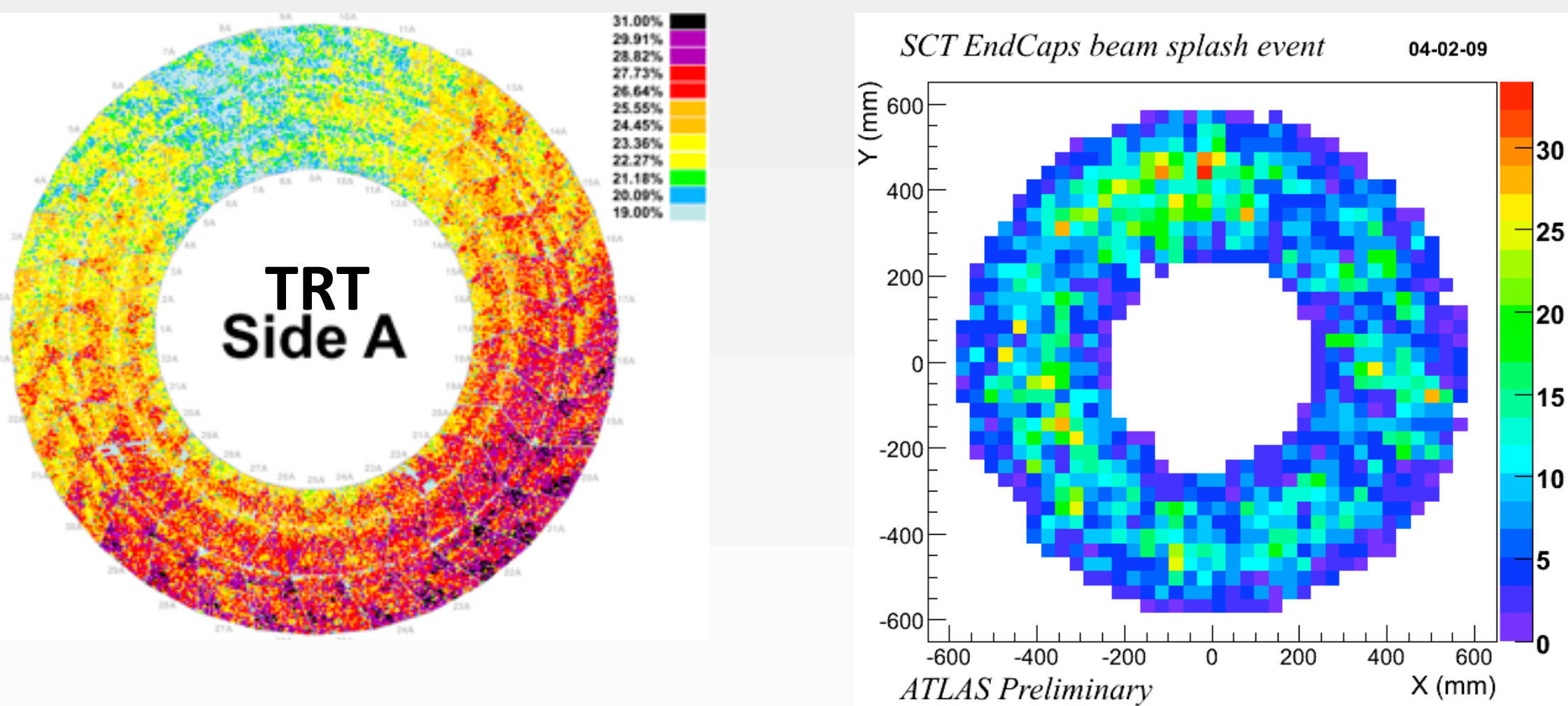


\section{Pixel performance}

- The charge distributions of clusters on tracks, with incidence angle close to the normal on the module, agree with expected

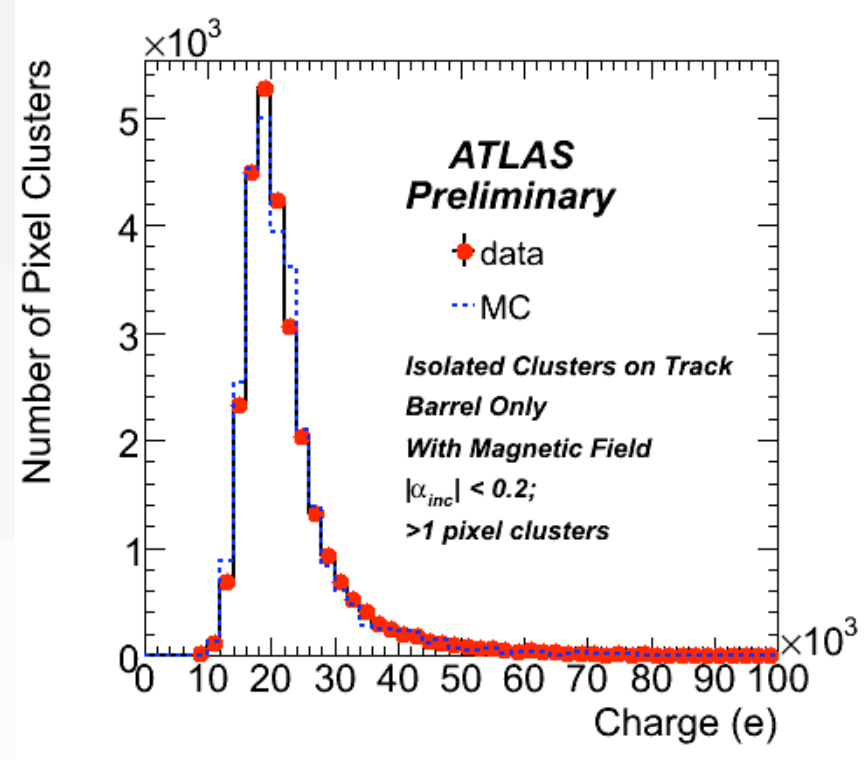

See J. Weingarten poster at this conference
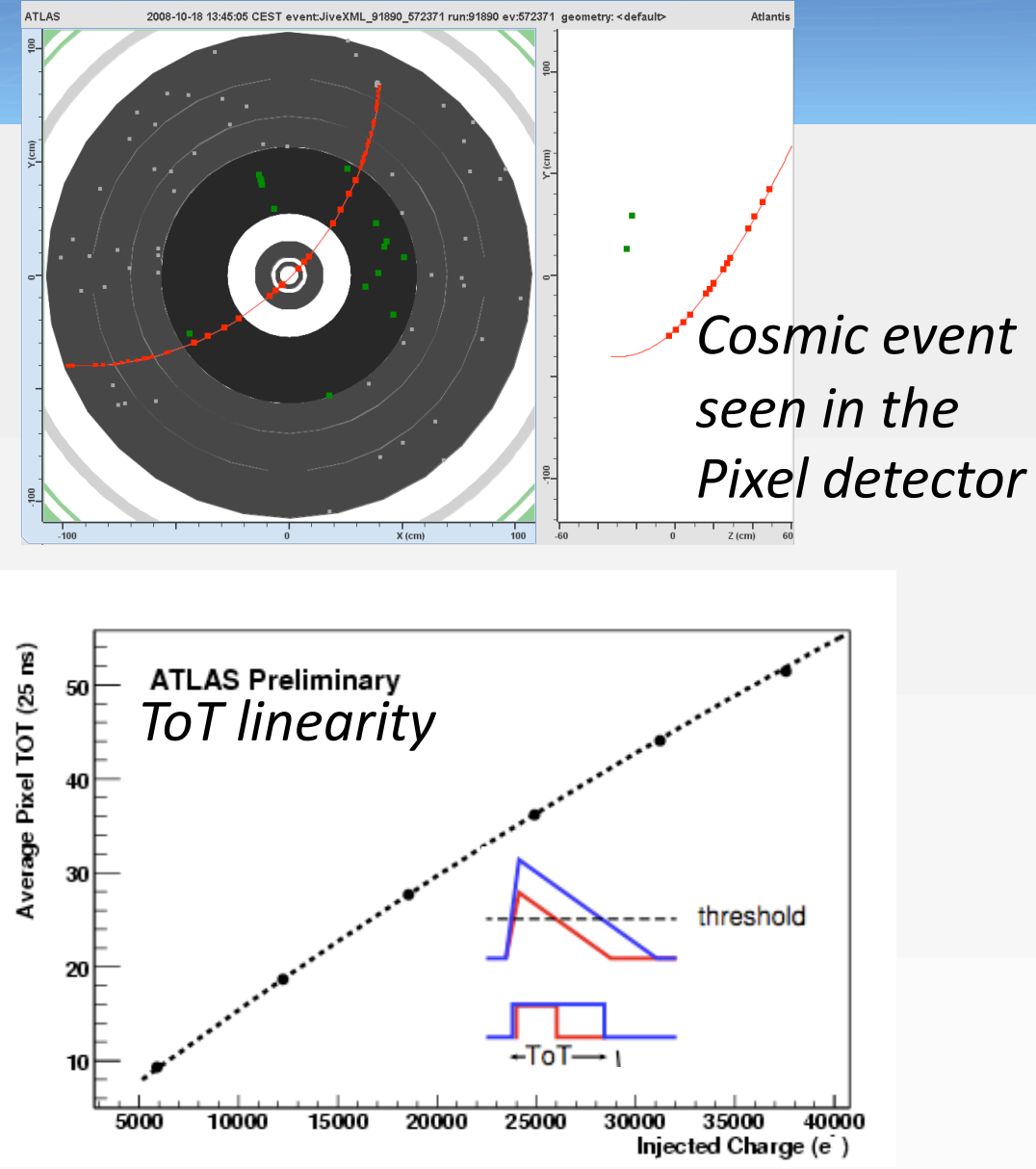

- The measured Time over Threshold (ToT) linear dependency on injected charge, used for charge calibration (BC unit) 


\section{SCT performance}

\section{Lorentz angle estimation}
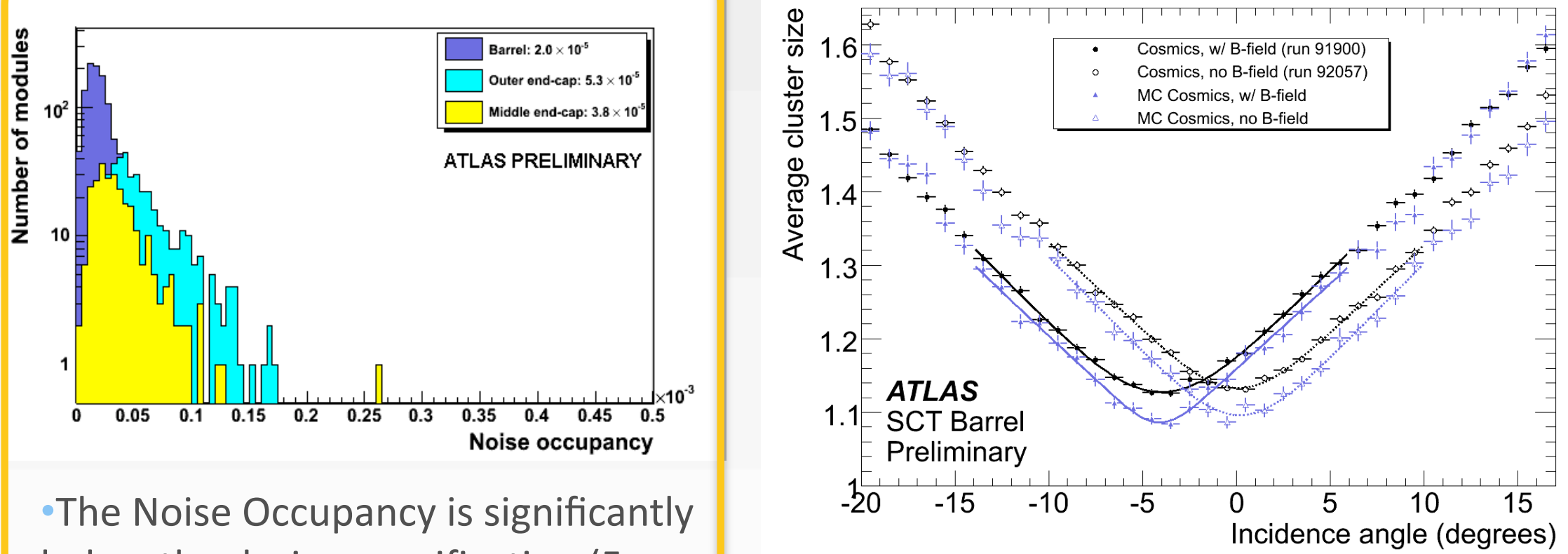

below the design specification ( $5 x$ $10^{-4}$, right edge of the plot)

- Cluster width versus track incident angle to the surface of the module (small discrepancy due to local temperature effects) 


\section{TRT performance}

- The turn-on of the production of TR photons as a function of gamma as measured for tracks of cosmic muons

- Different type of radiator materials:

- Barrel: polypropylene-polyethylene fiber mats ("chaotic" orientation of fibers/boundaries)

- End-caps: polypropylene foils ("aligned" orientation of boundaries)
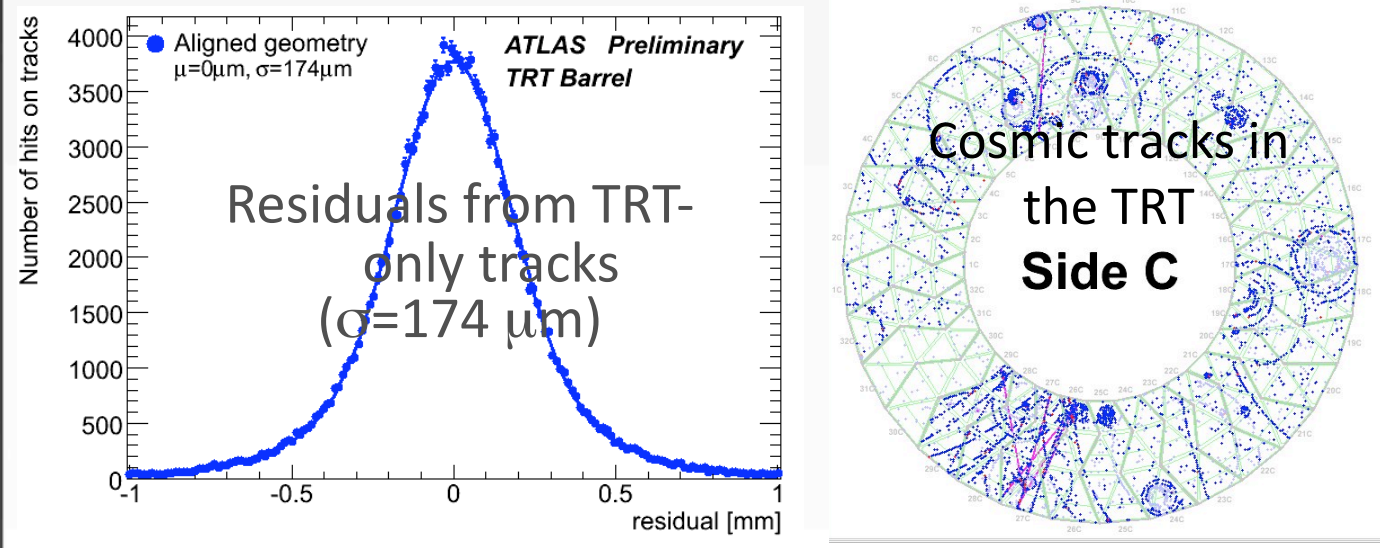

\section{Transition Radiation turn-on curves}
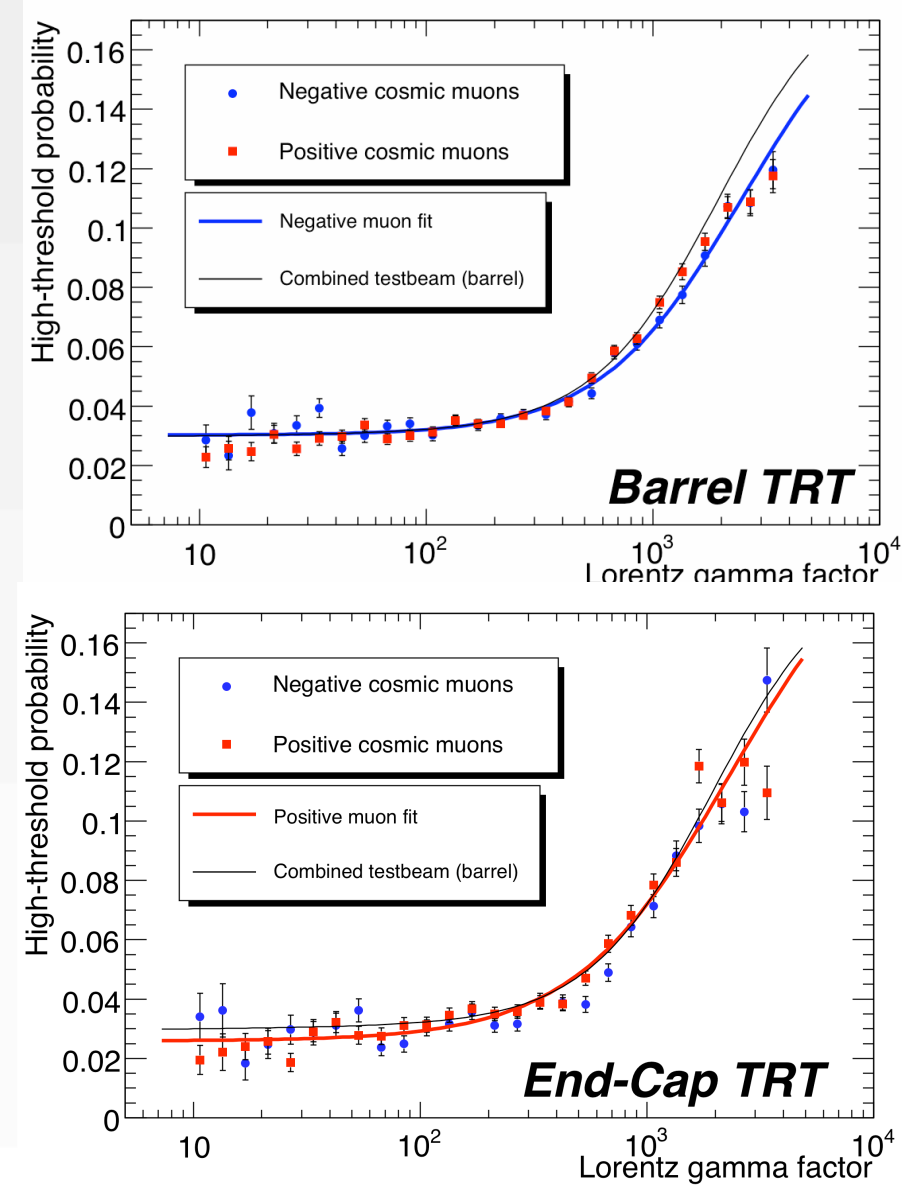


\section{ID combined tracking}
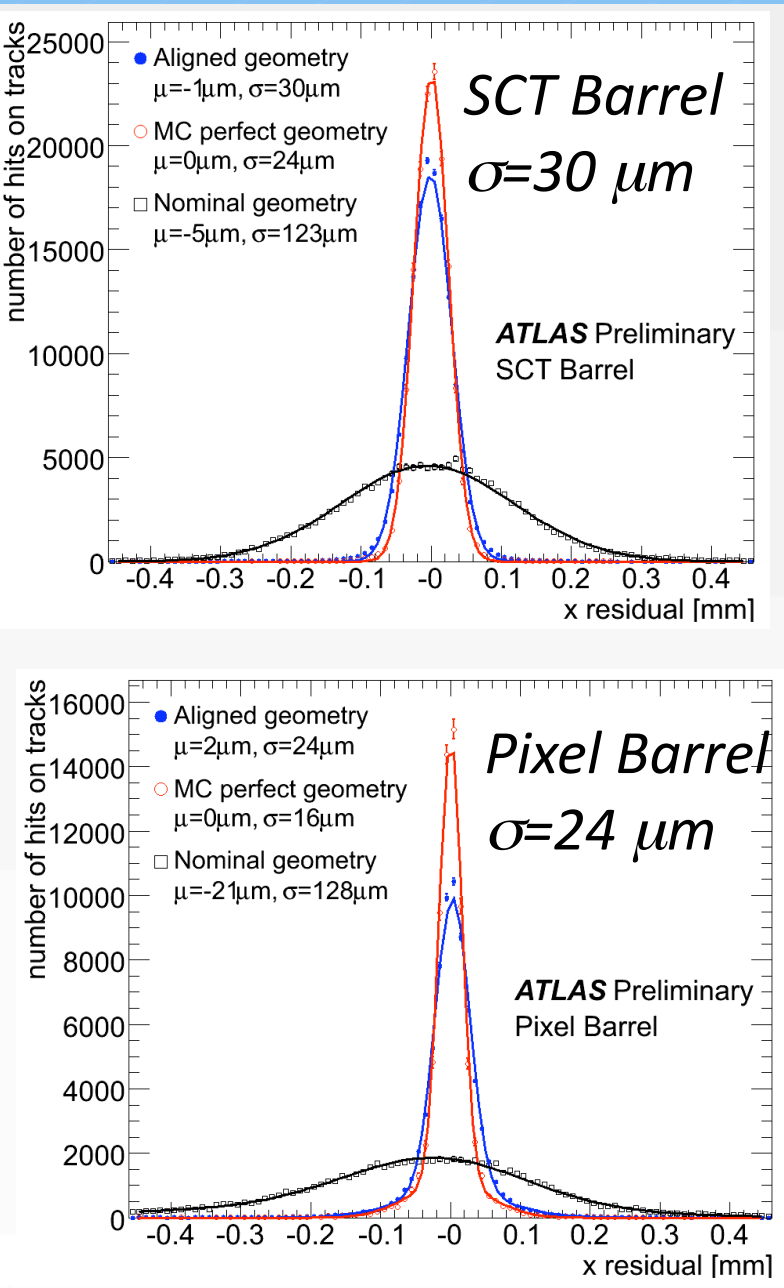

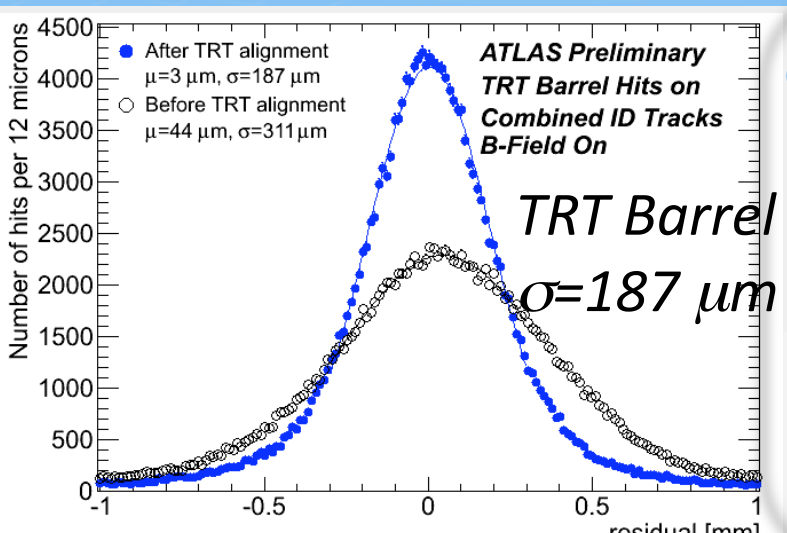

Inner detector tracking performance, before and after alignments

- The residual is defined as the measured hit position minus the expected hit position from the track extrapolation

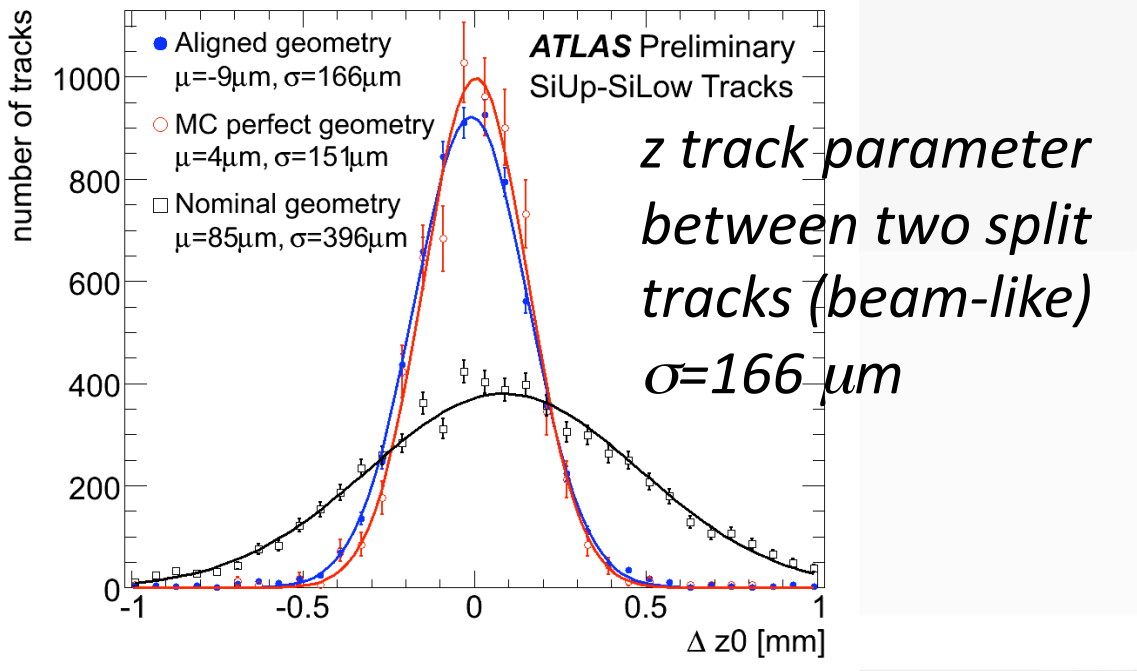




\section{ATLAS Combined performance}

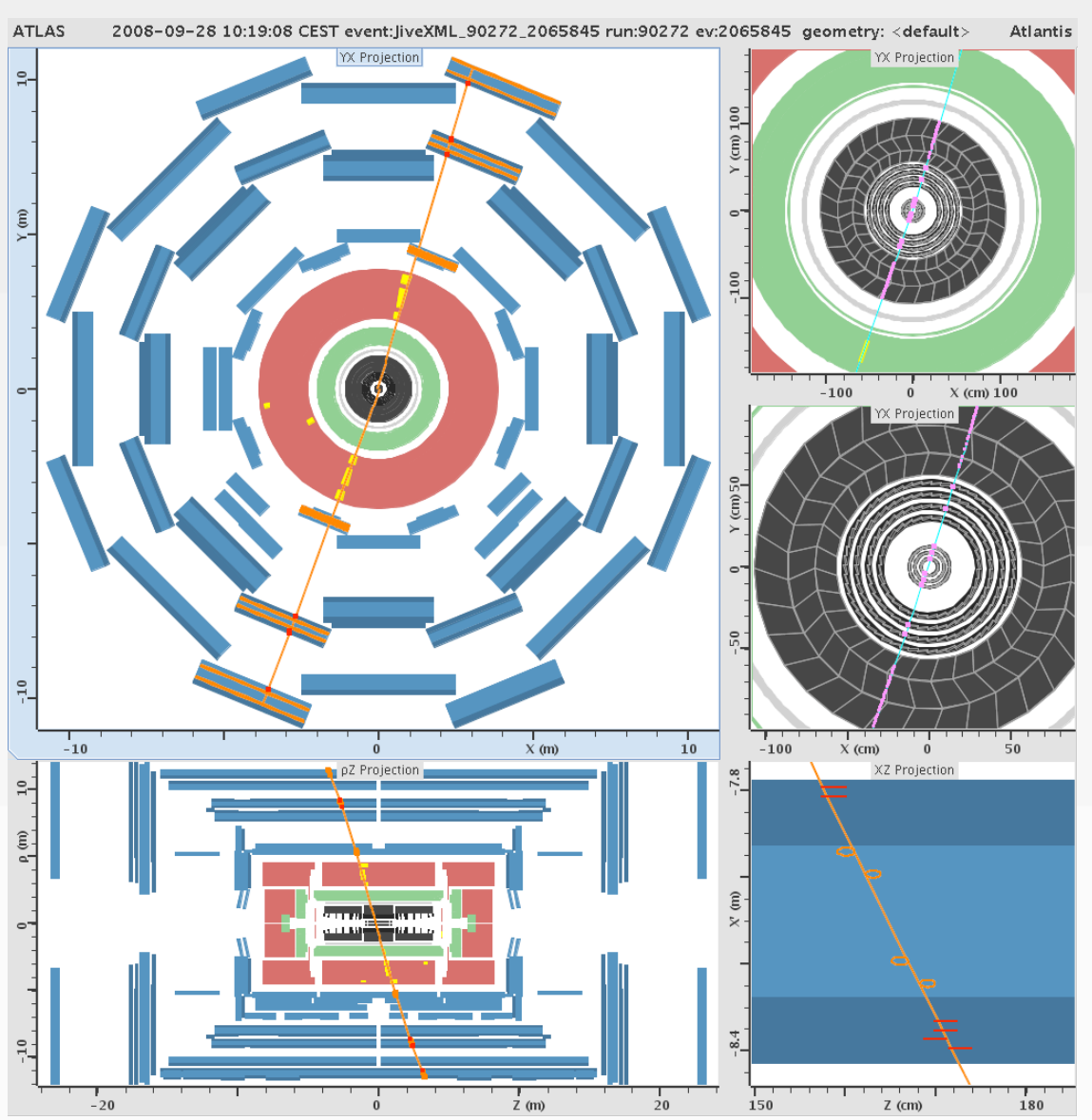

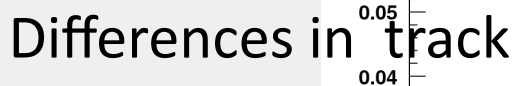
parameters in the muon spectrometer and the ID (preliminary alignment)

0 .

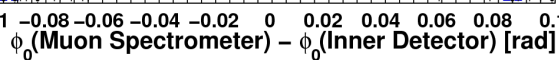

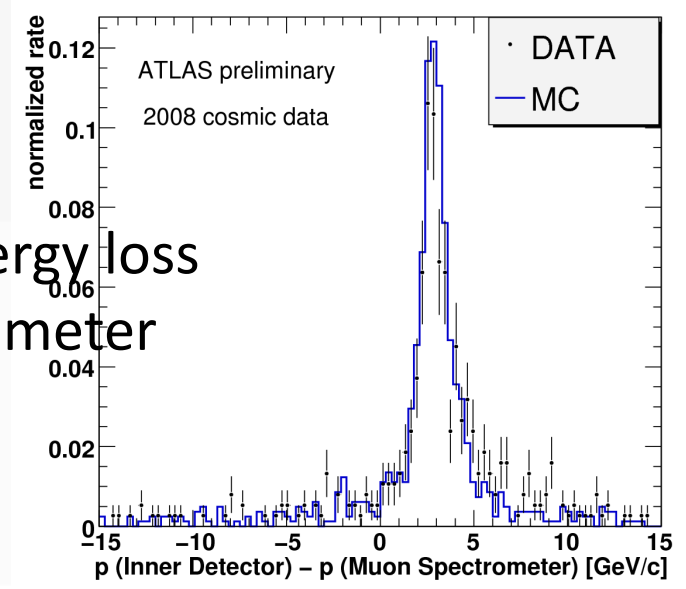




\section{Conclusions}

- ATLAS is a great challenge, due to the dimensions and the complexity of the detectors and to the discoveries it aims to reach

- The robustness and the stability of the dataflow are now under optimizations, with the final system completely integrated, and continuously exercised with cosmic rays

- Trigger systems are being prepared for different LHC scenarios and even the first beams will give important inputs to understand the detectors

- Up to now we were "going to", now we "are"!

- ...and to be honest, we are waiting for good news! 


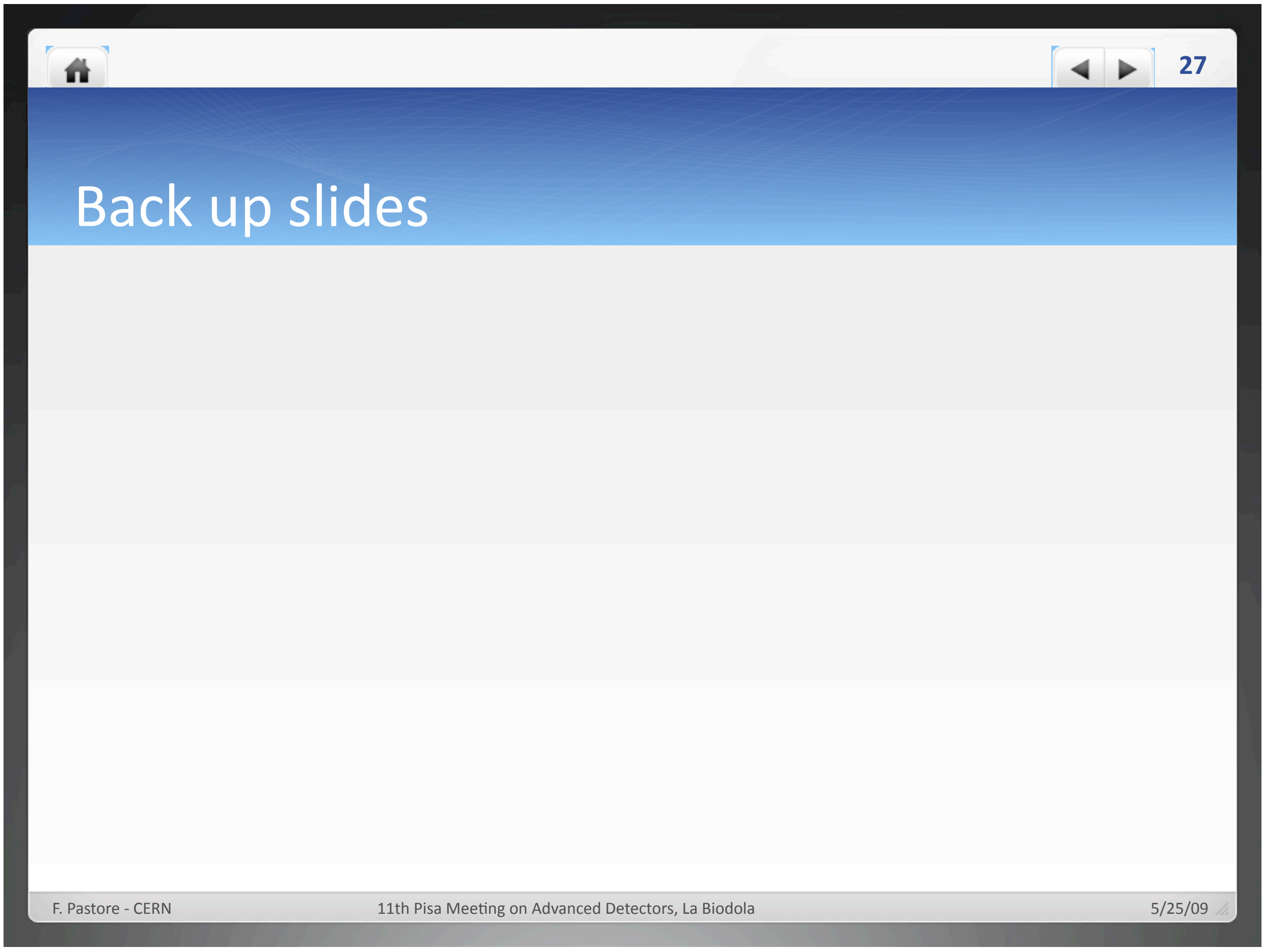




\section{LHC restart plan}

Year

Month

Baseline

Base
2009

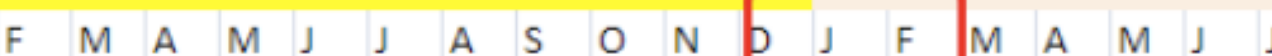

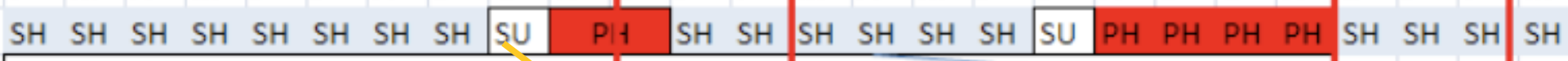
24 wreks physics pospible

$\mathrm{SH}$ SH SH SH SH SH SH SH $\mathrm{SU}$ PH PH PH PH PH PH PH PH PH PH PH SH SH SH SH SH SH 44 weeks physics pospible

- The machine will be cold by mid August, ready for first injected beam by second half of September

- Physics run at 5 TeV until Autumn 2010

- first physics run ( 10 months long) @10 TeV

- Heavy ion $\mathrm{Pb}-\mathrm{Pb}$ collision toward the end of the run

- Then complete installation of additional extra pressure relief valves on remaining dipole cryostat (essential for maximum energy operation)

- Restart in Spring 2011 with increasing beam entergergesess

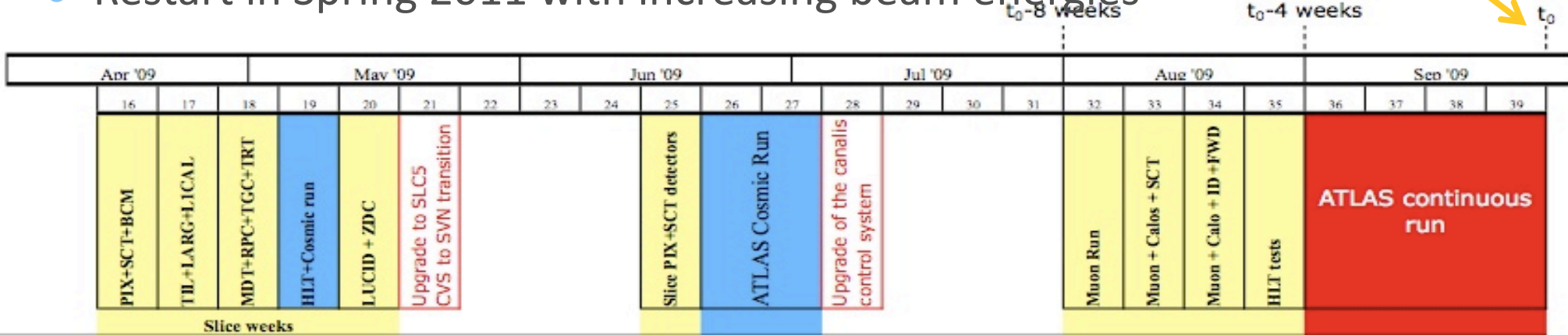

ATLAS run schedule 


\section{ATLAS Dataflow}

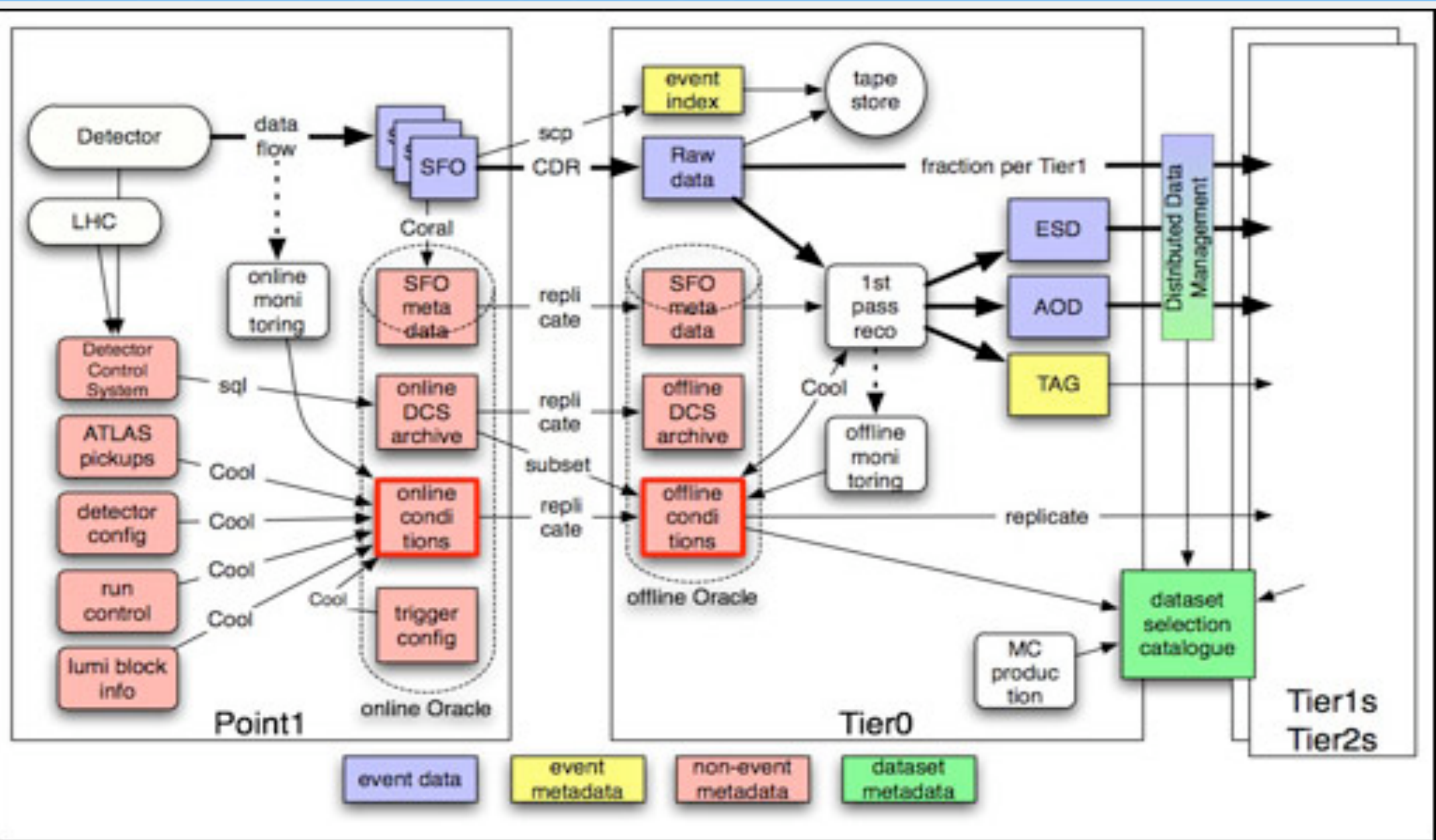

